\title{
Spectrum of turbulent Kelvin-waves cascade in superfluid helium
}

\author{
Andrew W. Baggaley圈 and Carlo F. Barenghi团 \\ ${ }^{1}$ School of Mathematics and Statistics, Newcastle University, Newcastle upon Tyne, NE1 7RU, England, UK
}

(Dated: October 17, 2018)

\begin{abstract}
To explain the observed decay of superfluid turbulence at very low temperature, it has been proposed that a cascade of Kelvin waves (analogous to the classical Kolmogorov cascade) transfers kinetic energy to length scales which are small enough that sound can be radiated away. We report results of numerical simulations of the interaction of quantized vortex filaments. We observe the development of the Kelvin-waves cascade, and compute the statistics of the curvature, the amplitude spectrum (which we compare with competing theories) and the fractal dimension.
\end{abstract}

PACS numbers: $\quad 67.25 . \mathrm{dk}, 47.37 .+\mathrm{q}$ 03.75.Kk

\section{MOTIVATION}

Quantum turbulence [1 [3] consists of a disordered tangle of reconnecting superfluid vortex filaments. Because of quantum mechanical constraints on the rotational motion, these filaments carry the same quantized (fixed) circulation $\Gamma$ and are very thin: the (fixed) vortex core radius, $a_{0}$, is much smaller than the average distance between the filaments, $\ell$. Quantum turbulence is easily created by agitating superfluid helium $\left({ }^{4} \mathrm{He}\right)$ with propellers [4], grids [5], forks [6] or wires [7], by applying a heat flow [8 [12] or by injecting a stream of ions [13]. It is also studied in superfluid ${ }^{3} \mathrm{He}-\mathrm{B}$ [14, 15] and, more recently, in atomic Bose-Einstein condensates [16, 17].

If the temperature is relatively large (more than $1 \mathrm{~K}$ in ${ }^{4} \mathrm{He}$ ), the turbulent kinetic energy contained in the superfluid vortices is transferred by the mutual friction [18] into the viscous gas of thermal excitations (the normal fluid) and then decays into heat; therefore a constant supply of energy (continuous stirring for example) is needed to maintain the intensity of the turbulence. If the temperature is relatively small (less than $1 \mathrm{~K}$ in ${ }^{4} \mathrm{He}$ ), the normal fluid is negligible, but, despite the absence of viscous dissipation, the turbulence still decays [19, 20]. The Kelvin-waves cascade [21 23] was proposed to explain this surprising effect.

A Kelvin wave is a rotating sinusoidal or helical displacement of the core of vortex filaments away from its unperturbed position [24 26]. The dispersion relation of a Kelvin wave of angular frequency $\omega$ and wavenumber $k$ along a straight vortex is [27]

$$
\omega=\frac{\Gamma}{2 \pi a_{0}^{2}}\left(1-\sqrt{1+\frac{K_{0}\left(k a_{0}\right)}{K_{1}\left(k a_{0}\right)}}\right),
$$

where $a_{0}$ is the vortex core radius and $K_{n}$ is the modified Bessel function of order $n$. In the long-wavelength approximation $\left(k a_{0} \ll 1\right)$, the angular frequency reduces to

$$
\omega \approx-\frac{\Gamma k^{2}}{4 \pi}\left(\ln 2 /\left(k a_{0}\right)-\gamma\right)=-\frac{\Gamma k^{2}}{4 \pi}\left(\ln 1 /\left(k a_{0}\right)-0.116\right)
$$

where $\gamma=0.5772$ is Euler's constant; the negative sign in this expression means that the Kelvin wave propagates in the direction opposite to the orientation of the unperturbed vorticity. Eq. 1 was originally derived for a thin, hollowcored vortex in a perfect Euler fluid; a similar expression was obtained for a vortex in a Bose-Einstein condensate [28, 29]. The simplified dispersion formula $\omega(k)=c k^{2}$, where $c$ is a constant, is often used in the Kelvin-waves cascade literature.

The Kelvin-waves cascade is the process in which the nonlinear interaction of Kelvin waves creates waves of shorter and shorter wavelength $\lambda=2 \pi / k$. At high enough temperature the mutual friction would quickly damp out the shorter Kelvin waves [27], but at low temperatures the cascade proceeds unhindered, until $k$ is large enough that sound is efficiently radiated away (phonon emission) by rapidly rotating vortices [30 33]. There is thus an analogy

\footnotetext{
*Electronic address: a.w.baggaley@ncl.ac.uk

${ }^{\dagger}$ Electronic address: c.f.barenghi@ncl.ac.uk
} 
between the Kelvin-waves cascade in quantum turbulence (where the energy sink is acoustic) and the Kolmogorov cascade [34, 35] in classical turbulence (where the energy sink is viscous).

It is thought that in superfluid helium at low temperatures both the Kolmogorov cascade and the Kelvin-waves cascades operate at the same time [36]. Let $\Lambda=L / V$ be the vortex line density (vortex length $L$ in the volume $V$ ) of a homogeneous isotropic vortex tangle, and $\ell \approx \Lambda^{-1 / 2}$ be the average intervortex spacing. The Kolmogorov cascade (which in a classical fluid is often described as the break up of eddies 37]) involves rearrangements or partial polarisation [38] of vortex lines over length scales of wavenumbers $k<<2 \pi / \ell$. The Kelvin-waves cascade occurs on individual vortex filaments for wavenumbers $k>>2 \pi / \ell$. Recent work has raised the possibility of the existence of a bottleneck [39, 40] between the two cascades, for which energy would pile up at some length scale of the order of $\ell$.

Direct evidence of the Kolmogorov cascade in superfluid helium was provided by the experiment of Maurer and Tabeling [41]. Numerical evidence is also convincing: the Kolmogorov $E_{k} \sim k^{-5 / 3}$ energy spectrum has been seen in calculations performed using both the Schwarz filament model [42] and the Gross-Pitaevskii equation [43-45] which describes a Bose-Einstein condensate. On the contrary, the Kelvin-waves cascade is less understood; there is no direct observation of it, and the theory is mired in a controversy between approaches proposed by L'vov, Nazarenko and collaborators [39, 40, 46 51] on one hand, and by Kozik and Svistunov [21, 33, 52 [56] on the other. The two theories predict similar Kelvin-waves amplitude spectra, but differ on matters of principles and in some important details.

Lacking direct experimental evidence of the Kelvin-waves cascade and its properties, experimentalists currently attempt to identify indirect but observable effects of the cascade and of any bottleneck; for example they study the temperature dependence of the observed effective kinematic viscosity [57, 58]. The results are still inconclusive.

The aim of our work at this stage is not to review or compare rival theories, but to add information. We present results of numerical calculations of the Kelvin-waves cascade. The few published calculations differ in details (e.g. whether the initial vortex is curved or straight, whether the cascade is forced continuously or results from an initial condition - either an artificially imposed cusp or a dynamically resolved vortex reconnection). More information is therefore necessary to learn the main physical features of the turbulent Kelvin-waves cascade and to assess which results are robust. In particular we shall focus the attention to the magnitude and spectrum of the Kelvin waves, the statistics of the vortex curvature and the fractal dimension.

Finally we note that, in presenting our results, we shall pay particular attention to how well energy is conserved in a given numerical approximations. In the absence of mutual friction, vortex evolution in between vortex reconnection events and without a turbulent cascade which radiates sound, energy should be conserved; yet previously published numerical calculations of the evolution of superfluid vortex filaments do not report how well the conservation law is satisfied.

\section{MODEL}

Hereafter we use parameters which refer to superfluid ${ }^{4} \mathrm{He}$ : circulation $\Gamma=9.97 \times 10^{-4} \mathrm{~cm}^{2} / \mathrm{s}$ and vortex core radius $a_{0} \approx 10^{-8} \mathrm{~cm}$, but our results can be generalised to turbulence in low temperature ${ }^{3} \mathrm{He}-\mathrm{B}$. Following Schwarz [59], we describe vortex filaments as space curves $\mathbf{s}=\mathbf{s}(\xi, t)$ where $\xi$ is arc length and $t$ is time. In the absence of mutual friction and of any externally applied superflow, the self-induced velocity of a superfluid filament at the point $\mathbf{s}$ is given by the Biot-Savart law

$$
\frac{d \mathbf{s}}{d t}=-\frac{\Gamma}{4 \pi} \oint_{\mathcal{L}} \frac{(\mathbf{s}-\mathbf{r})}{|\mathbf{s}-\mathbf{r}|^{3}} \times \mathbf{d r} \mathbf{r}
$$

The line integral extends over the entire vortex configuration $\mathcal{L}$, which is discretised into a large number of points $\mathbf{s}_{i}$ $(i=1, \cdots N)$. The singularity at $\mathbf{s}=\mathbf{r}$ is removed in a standard way by considering local and non-local contributions to the integral. If $\mathbf{s}_{i}$ is the position of the $i^{\text {th }}$ discretization point along the vortex line, Eq. 3 becomes 60 ]

$$
\frac{d \mathbf{s}_{i}}{d t}=\frac{\Gamma}{4 \pi} \ln \left(\frac{\sqrt{\ell_{i} \ell_{i+1}}}{a}\right) \mathbf{s}_{i}^{\prime} \times \mathbf{s}_{i}^{\prime \prime}-\frac{\Gamma}{4 \pi} \oint_{\mathcal{L}^{\prime}} \frac{\left(\mathbf{s}_{i}-\mathbf{r}\right)}{\left|\mathbf{s}_{i}-\mathbf{r}\right|^{3}} \times \mathbf{d r} .
$$

Here $\ell_{i}$ and $\ell_{i+1}$ are the arclengths of the curve between points $\mathbf{s}_{i-1}$ and $\mathbf{s}_{i}$ and between $\mathbf{s}_{i}$ and $\mathbf{s}_{i+1}$, and $\mathcal{L}^{\prime}$ is the original vortex line without the section between $\mathbf{s}_{i-1}$ and $\mathbf{s}_{i+1}$. For simplicity we assume that the cutoff parameter is $a \approx a_{0}$. Other de-singularization techniques are also used in the fluid dynamics literature 61, 62.

The number of discretization points, $N$, changes with time. As the simulation progresses, new discretization points are introduced to maintain the resolution along the vortex filament. If the separation between two points, $\mathbf{s}_{i}$ and 
$\mathbf{s}_{i+1}$, becomes greater than some threshold $\delta$ (which we call the minimum resolution), we introduce a new point at position $\mathbf{s}_{i^{\prime}}$ given by

$$
\mathbf{s}_{i^{\prime}}=\frac{1}{2}\left(\mathbf{s}_{i}+\mathbf{s}_{i+1}\right)+\left(\sqrt{R_{i^{\prime}}^{2}-\frac{1}{4} \ell_{i+1}^{2}}-R_{i^{\prime}}\right) \frac{\mathbf{s}_{i^{\prime}}^{\prime \prime}}{\left|\mathbf{s}_{i^{\prime}}^{\prime \prime}\right|}
$$

where $R_{i^{\prime}}=\left|\mathbf{s}_{i^{\prime}}^{\prime \prime}\right|^{-1}$. In this way $\mathbf{s}_{i^{\prime}}^{\prime \prime}=\left(\mathbf{s}_{i}^{\prime \prime}+\mathbf{s}_{i+1}^{\prime \prime}\right) / 2$, that is to say the insertion of new points preserves the curvature. Points are removed if their separation is less than $\delta / 2$, to ensure that our shortest length-scale is fixed. The above algorithms ensure that the separation of vortex points along a filament lies between $\delta / 2$ and $\delta$.

For accuracy, we approximate all spatial derivatives using $4^{\text {th }}$ order finite difference schemes which account for varying mesh sizes along the vortex filaments [63]. Let $\mathbf{s}_{i}$ be the $i^{\text {th }}$ point on the vortex filament; the two points behind have positions $\mathbf{s}_{i-2}$ and $\mathbf{s}_{i-1}$, and the two points in front have positions $\mathbf{s}_{i+1}$ and $\mathbf{s}_{i+2}$. We denote $\ell_{i-1}=\left|\mathbf{s}_{i-1}-\mathbf{s}_{i-2}\right|$, $\ell_{i}=\left|\mathbf{s}_{i}-\mathbf{s}_{i-1}\right|, \ell_{i+1}=\left|\mathbf{s}_{i+1}-\mathbf{s}_{i}\right|$, and $\ell_{i+2}=\left|\mathbf{s}_{i+2}-\mathbf{s}_{i+1}\right|$. We can then write

$$
\mathbf{s}_{i}^{\prime}=A_{1 i} \mathbf{s}_{i-2}+B_{1 i} \mathbf{s}_{i-1}+C_{1 i} \mathbf{s}_{i}+D_{1 i} \mathbf{s}_{i+1}+E_{1 i} \mathbf{s}_{i+2}
$$

where the coefficients $A_{1 i}, B_{1 i}, C_{1 i}, D_{1 i}$ and $E_{1 i}$ are given by,

$$
\begin{gathered}
A_{1 i}=\frac{\ell_{i} \ell_{i+1}^{2}+\ell_{i} \ell_{i+1} \ell_{i+2}}{\ell_{i-1}\left(\ell_{i-1}+\ell_{i}\right)\left(\ell_{i-1}+\ell_{i}+\ell_{i+1}\right)\left(\ell_{i-1}+\ell_{i}+\ell_{i+1}+\ell_{i+2}\right)} \\
B_{1 i}=\frac{-\ell_{i-1} \ell_{i+1}^{2}-\ell_{i} \ell_{i+1}^{2}-\ell_{i-1} \ell_{i+1} \ell_{i+2}-\ell_{i} \ell_{i+1} \ell_{i+2}}{\ell_{i-1} \ell_{i}\left(\ell_{i}+\ell_{i+1}\right)\left(\ell_{i}+\ell_{i+1}+\ell_{i+2}\right)} \\
C_{1 i}=-\left(A_{1 i}+B_{1 i}+D_{1 i}+E_{1 i}\right) \\
D_{1 i}=\frac{\ell_{i-1} \ell_{i} \ell_{i+1}+\ell_{i}^{2} \ell_{i+1}+\ell_{i-1} \ell_{i} \ell_{i+2}+\ell_{i}^{2} \ell_{i+2}}{\ell_{i+1} \ell_{i+2}\left(\ell_{i}+\ell_{i+1}\right)\left(\ell_{i-1}+\ell_{i}+\ell_{i+1}\right)} \\
-\ell_{i+1} \ell_{i}^{2}-\ell_{i-1} \ell_{i} \ell_{i+1} \\
E_{1 i}=\frac{\ell_{i+2}\left(\ell_{i+1}+\ell_{i+2}\right)\left(\ell_{i}+\ell_{i+1}+\ell_{i+2}\right)\left(\ell_{i-1}+\ell_{i}+\ell_{i+1}+\ell_{i+2}\right)}{\ell_{i+1}}
\end{gathered}
$$

In a similar manner we can write,

$$
\mathbf{s}_{i}^{\prime \prime}=A_{2 i} \mathbf{s}_{i-2}+B_{2 i} \mathbf{s}_{i-1}+C_{2 i} \mathbf{s}_{i}+D_{2 i} \mathbf{s}_{i+1}+E_{2 i} \mathbf{s}_{i+2}
$$

where the coefficients $A_{2 i}, B_{2 i}, C_{2 i}, D_{2 i}$ and $E_{2 i}$ are given by,

$$
\begin{gathered}
A_{2 i}=\frac{2\left[-2 \ell_{i} \ell_{i+1}+\ell_{i+1}^{2}-\ell_{i} \ell_{i+2}+\ell_{i+1} \ell_{i+2}\right]}{\ell_{i-1}\left(\ell_{i-1}+\ell_{i}\right)\left(\ell_{i-1}+\ell_{i}+\ell_{i+1}\right)\left(\ell_{i-1}+\ell_{i}+\ell_{i+1}+\ell_{i+2}\right)} \\
B_{2 i}=\frac{2\left[2 \ell_{i-1} \ell_{i+1}+2 \ell_{i} \ell_{i+1}-\ell_{i+1}^{2}+\ell_{i-1} \ell_{i+2}+\ell_{i} \ell_{i+2}-\ell_{i+1} \ell_{i+2}\right]}{\ell_{i-1} \ell_{i}\left(\ell_{i}+\ell_{i+1}\right)\left(\ell_{i}+\ell_{i+1}+\ell_{i+2}\right)} \\
C_{2 i}=-\left(A_{2 i}+B_{2 i}+D_{2 i}+E_{2 i}\right) \\
D_{2 i}=\frac{2\left[-\ell_{i-1} \ell_{i}-\ell_{i}^{2}+\ell_{i-1} \ell_{i+1}+2 \ell_{i} \ell_{i+1}+\ell_{i-1} \ell_{i+2}+2 \ell_{i} \ell_{i+2}\right]}{\ell_{i+1} \ell_{i+2}\left(\ell_{i}+\ell_{i+1}\right)\left(\ell_{i-1}+\ell_{i}+\ell_{i+1}\right)} \\
E_{1 i}=\frac{2\left[\ell_{i-1} \ell_{i}+\ell_{i}^{2}-\ell_{i-1} \ell_{i+1}-2 \ell_{i} \ell_{i+1}\right]}{\ell_{i+2}\left(\ell_{i+1}+\ell_{i+2}\right)\left(\ell_{i}+\ell_{i+1}+\ell_{i+2}\right)\left(\ell_{i-1}+\ell_{i}+\ell_{i+1}+\ell_{i+2}\right)}
\end{gathered}
$$


Note that if we set $\ell_{i-1}=\ell_{i}=\ell_{i+1}=\ell_{i+2}=h$, then the above expressions reduce to familiar finite-difference schemes for a uniform mesh:

$$
\begin{gathered}
\mathbf{s}_{i}^{\prime}=\frac{1}{12 h}\left(\mathbf{s}_{i-2}-8 \mathbf{s}_{i-1}+8 \mathbf{s}_{i+1}-\mathbf{s}_{i+2}\right)+\mathcal{O}\left(h^{4}\right), \\
\mathbf{s}_{i}^{\prime \prime}=\frac{1}{12 h^{2}}\left(-\mathbf{s}_{i-2}+16 \mathbf{s}_{i-1}-30 \mathbf{s}_{i}+16 \mathbf{s}_{i+1}-\mathbf{s}_{i+2}\right)+\mathcal{O}\left(h^{4}\right) .
\end{gathered}
$$

The Biot-Savart law describes an incompressible flow. In order to model energy dissipation at large $k$ (phonon emission), we also remove points if the local wavelength is smaller than a specified value. Since the smallest lengthscale is $\delta / 2$, the maximum curvature is of the order of $2 / \delta$. At each time step we compute the local curvature $C(\xi)=\left|\mathbf{s}^{\prime \prime}\right|=\left|d^{2} \mathbf{s} / d \xi^{2}\right|$ at each discretization point $\mathbf{s}_{j}(j=1, \cdots N)$ If, at some location along a vortex filament, the local curvature exceeds the critical value $1.9 / \delta \mathrm{cm}^{-1}(95 \%$ of the maximum value $2 / \delta)$, points are removed and the filament is smoothed locally. This leads to a loss of line length, hence a loss of energy.

It must be stressed that we are not interested in the phonon emission per se, but rather in the consequence of the presence of an energy sink on the Kelvin waves cascade. The actual numerical value of $\delta$ is chosen for numerical convenience and is orders of magnitude larger than the microscopic lengthscales which are physically relevant to phonon emission and vortex reconnections. Therefore our model contains (and controls) a mechanism of dissipation, and is different in scope from vortex methods dedicated to perfect Euler dynamics. In the next section we describe results obtained with two different spatial resolutions: $\delta=10^{-3} \mathrm{~cm}$ (high resolution) and $\delta=5 \times 10^{-3} \mathrm{~cm}($ low resolution). We shall see that our results are independent of the resolution used.

Time evolution is based on a $3^{\text {rd }}$ order Adams-Bashforth scheme. Consider an evolution equation of the form $d \mathbf{s} / d t=\mathbf{v}$. We time-step the equation according to

$$
\mathbf{s}_{i}^{n+1}=\mathbf{s}_{i}^{n}+\frac{\Delta t}{12}\left(23 \mathbf{v}_{i}^{n}-16 \mathbf{v}_{i}^{n-1}+5 \mathbf{v}_{i}^{n-2}\right)+\mathcal{O}\left(\Delta t^{4}\right)
$$

where $\Delta t$ is the time step and the superscript $n$ refers to the time $t_{n}=n \Delta t(n=0,1,2, \cdots)$. Lower order schemes are used for the initialisation, when older velocity values are not available. The time step $\Delta t$ is chosen so that the period $\tau_{\min }=2 \pi / \omega_{\max }$ of the fastest Kelvin wave (which has wavenumber $k_{\max }=2 \pi / \lambda_{\min } \approx \pi / \delta$ ) contains at least 25 time steps; we have verified that reducing our time step further gives the same results.

If two discretization points become closer to each other than the local discretization distance, our numerical algorithm reconnects the two filaments 64] subject to the criteria that the total length (as proxy for energy) decreases [65]. In this way the same quantity $\delta$ controls both energy dissipation mechanisms in our model - sound emission and reconnections. Self-reconnections (which can arise if a vortex filament has twisted and coiled by a large amount) are treated in the same way. Since reconnections involve only anti-parallel filaments, prior to reconnection we form local (unit) tangent vectors $\mathbf{s}^{\prime}=d \mathbf{s} / d \xi$, where $\xi$ is arc length, and, using the inner product, we check that the two filaments are not parallel. Finally, We remove any small loops with less than three discretization points.

During the evolution we monitor the length $L$ and the kinetic energy $E$ (in unit of the density) of the vortex configuration; the latter is

$$
E=\frac{1}{2} \int_{V} \mathbf{v}^{2} d V
$$

If the calculation is performed in a periodic box, the vortices can extend across the boundaries; the only way to compute the energy integral is to discretise the integrand on a three-dimensional spatial grid, computing the velocity at each grid point; unfortunately this procedure converges too slowly to be practical. However, if the calculation is performed in an infinite volume, the vortex filaments are closed loops. Assuming that the velocity field decays to zero at infinity, the energy integral can be transformed into a line integral [66]

$$
E=\frac{1}{2} \int_{V} \mathbf{v}^{2} d V=\int_{V} \mathbf{v} \cdot \mathbf{r} \times \boldsymbol{\omega} d V=\Gamma \oint_{\mathcal{L}} \mathbf{v} \cdot \mathbf{r} \times \mathbf{s}^{\prime} d \xi
$$

which converges much better. In writing Eq. 22 we have used the fact that the vorticity $\boldsymbol{\omega}=\nabla \times \mathbf{v}$ is concentrated on the vortex filaments $\left(\boldsymbol{\omega} d V=\Gamma \mathbf{s}^{\prime} d \xi\right)$.

In the absence of mutual friction, during Biot-Savart evolution (but not during vortex reconnections, and not while the Kelvin-waves cascade is in progress with the associated sound emission which we model), the kinetic energy $E$ 
is conserved, hence changes in $E$ measure the accuracy of the numerical method. Our current method conserves energy about a factor of ten better than our preliminary unpublished calculations. The energy-conserving properties of previous calculations in the superfluid literature is unknown.

Unlike the energy $E$, the vortex length $L$ is not conserved. However, if the Biot-Savart law is replaced by the Local Induction Approximation (LIA), the length is conserved. The LIA was used in the original work of Schwarz [59] and by others to reduce the computational cost. We do not use the LIA in our calculations.

A quantity which is particularly interesting is the normalized histogram (probability density function, of PDF for short) of the curvature $C(\xi)$, sampled on the discretization points: we call this quantity $\operatorname{PDF}(C)$.

\section{RESULTS}

\section{A. Cascading and non-cascading vortex configurations}

In the first set of numerical experiments we consider a single vortex filament set across a periodic box as in Fig. 11. The initial shape of the vortex is not straight, but is perturbed by two sinusoidal Kelvin waves of amplitude $A$ and different wavelengths $\lambda$. We compute the time evolution and observe that that this initial condition does not yield a cascade to shorter waves. However, if we start with three parallel vortex filaments (each filament with two Kelvin waves), as shown in Fig. 2 (left), we notice that, as the vortices rotate around each other, they become more wiggly, see Fig. 2(right).

This result suggests that, besides vortex reconnection events (as shown in Ref. [22]), the Kelvin-waves cascade can be initiated, more in general, by the interaction of nearby curved vortices via the velocity field which they induce on each other. The result is also consistent with wave-turbulence theory [67], which states that, for one-dimensional waves obeying a dispersion law of the form $\omega(k)=c k^{2}$, a four-waves process is not sufficient to generate a cascade. According to the theory, the minimal initial condition for the cascade consists of three waves which satisfy the resonant condition that $k_{1}+k_{2}+k_{3}=k_{4}+k_{5}+k_{6}$ and $\omega\left(k_{1}\right)+\omega\left(k_{2}\right)+\omega\left(k_{3}\right)=\omega\left(k_{4}\right)+\omega\left(k_{5}\right)+\omega\left(k_{6}\right)$, where indices $1,2,3$ and 4,5,6 refer to incoming and outgoing waves respectively. The initial condition shown in Fig. 2(left) contains more waves than the minimal number of waves (if it contained only the minimal number of waves we would need to seek the exact resonant condition, which is not our purpose at this stage).

In order to pursue this investigation more quantitatively, we want to monitor the kinetic energy of the vortex configuration. Unfortunately, as we have mentioned in Section [II] if the vortex configurations is set in a periodic box, it is not possible to accurately determine its energy. Hereafter, in order to use Eq. 22, we consider only closed vortex loops contained in an infinite volume.

In a second set of numerical experiments we start with a single vortex ring in an infinite volume; the ring is seeded with two Kelvin waves, as shown in Fig. 3. During the time evolution we observe that the waves interact, but there is no cascade, consistently with

the evolution of the vortex line of Fig. 1. Indeed, if we examine the PDF of the curvature, we notice that $\operatorname{PDF}(C)$ does not evolve in time to larger values of $C$, as shown in Fig. 4 (top). Unlike the vortex line in the periodic box of Fig. 1. now we can monitor how well the kinetic energy is conserved in our numerical calculations. Fig. 4(bottom) shows that the relative change of energy during the time evolution of this non-cascading vortex configuration of interacting Kelvin waves is only $\Delta E / E \approx \pm 0.06$ percent. This value describes how well we can integrate Euler dynamics.

\section{B. Curvature and energy}

Proceeding in analogy with Fig. 2 we now consider a third set of numerical experiments with three vortex rings oriented in the same direction as shown in the first snapshot of Fig. 5 . each ring is initially perturbed by two random Kelvin waves. This set-up contains enough waves to trigger the Kelvin-waves cascade and allows us to carefully monitor the energy.

The initial total number of discretization points is $N_{0}=600$; at the end of the calculation at time $t=2 \mathrm{~s}$ we have $N=773$. The minimum distance between discretization points is set to $\delta=5 \times 10^{-4} \mathrm{~cm}$. All three rings are oriented in the same way, hence they travel in the same (negative $z$ ) direction, leapfrogging around each other. This simple vortex bundle configuration evolves into a Kelvin-waves cascade: Fig. [5 shows that during the evolution the wiggliness of the vortices increases. This is in agreement with the straight vortices of Fig. 2 Since the wiggly vortices of Fig. 5 are relatively close to each other, there are many reconnections up to time $t=0.1 \mathrm{~s}$, less reconnections between $0.1<t<0.5 \mathrm{~s}$, and no reconnection afterwards. The reconnections do not appear to affect the vortex configuration in a significant way: the vortex bundle seems structurally robust. 
We start our analysis of the Kelvin-waves cascade by noticing that it induces a stretching of the vortices: Fig. 6 shows that the relative increase of vortex length is $\Delta L / L \approx 6 \%$. This effect (the transformation of part of the interaction energy into length) is in agreement with previous calculations of reconnecting vortex bundles performed using the Gross-Pitaevskii model [68, 69].

The behaviour of the energy $E$ is shown in Fig. 7 During the initial stage of the evolution $(0<t<0.5 \mathrm{~s})$ the energy slightly declines, then it becomes approximately constant, with relative fluctuations $\Delta E / E$ of less than $3 \%$. The energy fluctuations are larger than for non-cascading vortex configurations, because in the cascading case the presence of short, rapid Kelvin waves induces rapid velocity fluctuations. Fig. 8 (top) and Fig. 8 (bottom) show respectively the maximum speed $v_{\max }$ and the maximum acceleration $(d v / d t)_{\max }$ sampled over the discretization points $\mathbf{s}_{j}(j=1, \cdots N)$. It is apparent that velocity fluctuations are larger during the initial stage of the cascade $(0<t<0.5 \mathrm{~s})$. We shall return to why the energy initially decreases after discussing the vortex curvature.

Fig. 9 shows the time evolution of mean, maximum and minimum curvature, $\langle C\rangle, C_{\max }$ and $C_{\min }$, sampled over the discretization points $\mathbf{s}_{j}$. Fig. 9(top) shows that $\langle C\rangle$ undergoes a rapid initial rise, then slowly decays, almost settling down. Fig. 9(bottom) shows that the maximum curvature is always much less than the maximum value of curvature allowed by the numerical resolution, which is $1900 \mathrm{~cm}^{-1}$ and is represented by the dashed line. The curvature $\mathrm{PDF}$ is shown in Fig. 10 Note that, as time progresses, $\operatorname{PDF}(C)$ moves to the right towards larger values of $C$. This effect must be compared against the much smaller movement to the right of the curvature PDF for the non-cascading vortex configuration which is showed in Fig. 团(top). Numerical experiments suggest that PDF $(C)$ is indeed a convenient quantity to monitor if one looks for evidence of the Kelvin-waves cascade. At later stages, as energy is lost at small scales, we observe that the turbulence decays, as in the experiments [19, 20], and the curvature PDF moves to smaller values of $C$.

It is also interesting to note in Fig. 10 that, after an initial transient, the curvature PDF assumes a power law dependence $\operatorname{PDF}(C) \sim C^{\alpha}$ for large values of $C$. Fig. 11 shows that the exponent $\alpha$ quickly settles down to the average value $\alpha \approx-3.5$. In the low resolution run $\left(\delta=10^{-3} \mathrm{~cm}\right)$ a similar calculation gives $\alpha=-3.34$, which confirms that our results are independent of the numerical resolution.

We can now return to the question as to why there is a small energy loss during the initial stage of the evolution from $t=0$ to $t \approx 0.5 \mathrm{~s}$ in Fig. 7 Our numerical algorithm has two mechanisms through which length, hence energy, can be lost: vortex reconnections and smoothing of regions of high curvature. Which is more important? To find the answer we repeat the calculation with the vortex reconnection algorithm switched off. The results are shown in Fig. 12, If we compare the energy computed with reconnections (Fig. 7) and without reconnections (Fig. 12(top)) we see that $E$ is more constant without reconnections. It is also instructive to compare curvature statistics computed with reconnections (Fig. 9) and without reconnections (Fig. 12 bottom three graphs). It is apparent that vortex reconnections are the most important energy sink at large $k$ : without reconnections, the maximum curvature $C_{\max }$ is much larger, and often reaches the numerical limit represented by the horizontal dashed line in Fig. 12(bottom). Since $C_{\min }$ is approximately the same, $\langle C\rangle$ is also larger than in the reconnecting case. Therefore, without vortex reconnections the cascade proceeds to the smallest scale which is numerically allowed, at which point the smoothing algorithm kicks in. We conclude that the initial energy loss shown in Fig. [7 is due to the frequent reconnections which occur during the initial stage of the evolution.

\section{Spectrum}

The next quantity of interest is the amplitude $a(\xi)$ of the Kelvin waves and the spectrum of the Kelvin-waves cascade. Following Svistunov [21], we introduce the concept of smoothed vortex filament: at every time $t$, we use every $n=15$ points as nodes of a cubic-spline interpolation to obtain a new (smoothed) filament, $\mathbf{s}_{\text {smooth }}$ from the original discretised vortex filament $\mathbf{s}_{j}(j=1, \cdots N)$. Fig. 13 (top) is an example of this procedure: in the figure we see the actual discretization points $\mathbf{s}_{j}(j=1, \cdots N)$ together with the smoothed line. We choose $n=15$ because, in a range which is approximately $10<n<20$, the amplitude spectrum (which we shall define shortly) does not depend on $n$, as shown in Fig. 13(bottom), confirming that our numerical smoothing procedure is robust enough.

We then define the Kelvin wave amplitude $a(\xi)$ as the distance between the original filament and the smoothed filament: $a(\xi)=\left|\mathbf{s}-\mathbf{s}_{\text {smooth }}\right|$. The mean amplitude $\langle a\rangle$ as a function of time is shown in Fig. 14. Note that the mean amplitude $\langle a\rangle$ saturates for $t>1 \mathrm{~s}$.

Finally, the amplitude spectrum, $A(k)$, is defined as

$$
\frac{1}{2} \int a^{2}(\xi) d \xi=\int_{0}^{\infty} A\left(k^{\prime}\right) d k^{\prime}
$$

and is shown in Fig. 15 We find that the amplitude spectrum behaves as $A(k) \sim k^{\beta}$ for large $k$. The best fit is 
$\beta=-3.10$; we obtain $\beta=-3.21$ in the low resolution run. Fig. 16] shows that the scaling exponent $\beta$ is independent of time, as the Kelvin-waves cascade develops.

\section{Angle}

From the dot product of the local tangent vectors, $\mathbf{s}^{\prime}$ and $\mathbf{s}_{\mathrm{smoth}}^{\prime}$, at each time $t$ we calculate the angle $\theta$ between the original vortex filament $\mathbf{s}$ and the smoothed filament $\mathbf{s}_{\mathrm{smooth}}$. The normalised histogram, $\mathrm{PDF}(\theta), \operatorname{computed}$ at $t=0.002 \mathrm{~s}$ is shown in Fig. [17(top). The peak value is approximately at $\theta \approx 15^{\circ}$.

In order to compare the distribution which we find with that of a standard normal distribution (zero mean and unit standard deviation), we make use of the following standard result from statistics. All normal random variables with mean $\mu$ and variance $\sigma$ can be 'standardised' to fit a standard normal distribution by subtracting the mean and scaling this quantity by the standard deviation. Fig. (17) (bottom) shows a plot of $z_{\theta}=(\theta-\bar{\theta}) / \sigma(\theta)$ where $\bar{\theta}$ and $\sigma(\theta)$ are respectively the mean and the variance of the distribution of the angle $\theta$. It is apparent that the distribution of the angles is close to a normal distribution. This result validates the weak-turbulence theory approach to the Kelvin-waves cascade problem [0]. A small departure from Gaussianity is present at large angles; it is likely that this effect is due to the fractal nature of the vortex filament (see section IIIF).

\section{E. Reconnecting vortex rings}

The last set of numerical experiments is concerned with two vortex rings which reconnect with each other. This configuration is similar to the collision of four vortex rings which was used in a previous work [22]. Fig. 18] shows the time evolution. It is apparent that, over the time scale which is considered, no Kelvin-waves cascade develops. This visual conclusion is confirmed by the inspection of $\operatorname{PDF}(C)$, shown in Fig. 19)(top), which does not progress in time to larger values of $C$, unlike Fig. 10. Fig. 19 (bottom) shows that the energy remains constant within $\Delta E / E \sim \pm 0.12 \%$.

If the initial vortex rings are not perfectly circular, however, the evolution proceeds toward a Kelvin-waves cascade, as shown in Fig. 20, The increasing wiggliness of Fig. 20 must be compared against Fig. 18, Following the initial reconnection of the two rings, we observe 4 more reconnections, which are actually self-reconnections since there is only one vortex filament left. Fig. 20(c) shows a self-reconnection event which arises from a large twisting (supercoiling) of the vortex filament, and which results in the emission of a small vortex loop. This self-crossing scenario was predicted by Kozik and Svistunov[55]. Similar small-loop emission were also observed in numerical studies of vortex bundle dynamics [68].

The PDF of the curvature confirms the existence of a cascade: Fig. 211(top) shows that PDF $(C)$ progresses in time to larger values of $C$. Fig. 21 (bottom) shows the behaviour of the energy, which has the same slight initial decreases as in Fig. 7 which we have discussed.

\section{F. Fractal dimension}

To quantify the wiggliness of the vortex configuration undergoing the Kelvin-waves cascade, we compute the correlation dimension $c_{D}$ introduced by Grassberger and Procaccia 71]. The correlation dimension provides an upper-bound estimate of the fractal dimension The definition is the following: given the $N$ discretization points $\mathbf{s}_{j}(j=1, \cdots N)$, we define the correlation integral as $K(\epsilon)=n / N^{2}$ where $n$ is the number of points whose separation is less than $\epsilon$. In the limit of $\epsilon \rightarrow 0, K(\epsilon)$ takes the form,

$$
K(\epsilon) \sim \epsilon^{c_{D}}
$$

where $c_{D}$ is the correlation dimension. This quantity can be calculated efficiently at the same time as the Biot-Savart integral is calculated. The result is shown in Fig. 22 for reconnecting vortex rings undergoing the Kelvin-waves cascade. Note the rapid increase of $c_{D}$ during the initial stage caused by reconnection events, followed by a decline due to the numerical dissipation at high wavenumbers which models physical dissipation (sound emission).

\section{DISCUSSION}

Our numerical calculations show that the Kelvin-waves cascade can be initiated by the interaction of neighbouring vortex lines as well as by vortex reconnections. We find that, shortly after an initial transient, the Kelvin-waves 
cascade quickly settles in and saturates: Fig. (11) and Fig. (16) show that the slopes $\alpha$ and $\beta$ of the curvature histogram at large $C, \operatorname{PDF}(C) \sim C^{\alpha}$, and of the amplitude spectrum at large $k, A(k) \sim k^{\beta}$, remain approximately constant.

We also find that the Kelvin-waves amplitude spectrum scales approximately as $A(k) \sim k^{-3.1}$ for large $k$, in agreement with $A \sim k^{-3}$ reported in Ref. 23] for a continuously excited vortex line, and less steep but in fair agreement with the theories of L'vov, Nazarenko and collaborators [51] and of Kozik and Svistunov [56], who predict $n_{k} \sim k^{-11 / 3}=k^{-3.7}$ and $n_{k} \sim k^{-17 / 5}=k^{-3.4}$ respectively, where their kelvon occupation number $n_{k}$ is equivalent to our $A(k)$. The applicability of wave turbulence theory to the problem is supported by our finding that the angle $\theta$ between the smoothed vortex line and the actual line is small and has approximately a Gaussian nature, see Fig. 17.

The regime which we have identified in our calculation is clearly a weak turbulent regime, as demonstrated by the small angles between the smoothed vortex filament and the actual filament - see Fig. 17(top). We think that in a stronger regime larger twists would appear and induce more self-reconnections than the few which we observe in Fig. 20. In must be stressed that our numerical cutoff effectively short-circuits the formation of loops at scales smaller than $\delta$. The tendency of the vortex filaments to twist could be explored more in detail by examining the torsion, but we refrain from this calculation due to larger errors which would arise in computing third derivatives. A better approach would be to model the cascade more microscopically using the Gross-Pitaevskii equation, although the range of scales which would be available is limited.

The possibility that the geometry of superfluid turbulence undergoes a qualitative change at low temperatures, where the friction can be ignored, was first considered by Tsubota and collaborators [72]. They noticed that if a vortex tangle is computed in the absence of the smoothing effect of the mutual friction, its appearance is "kinky". Further work 73. showed that, even in the presence of a small amount of friction, the vortex tangle is a fractal object. This result stimulated the study of the relation between energy and length, and between energy and fractal dimension [74 76]. The results which we present here show that, in its simplest form, the fracture nature of superfluid turbulence arises from the Kelvin-waves cascade along individual filaments. The actual value of the fractal dimension depends on the level of acoustic dissipation of kinetic energy, which in our Biot-Savart simulations is modelled by the numerical dissipation via the parameter $\delta$. Therefore, for decaying turbulence as in our calculations, $c_{D}$ depends on time. The fact that we observe some secondary reconnections only in the evolution shown in Fig. 20 means that probably fractality is a general feature of the Kelvin-waves cascade, not the result of a sequence of self-similar twisting and secondary reconnections; it would be interesting to pursue this aspect of the problem with more computing power and a smaller $\delta$. It is worth remarking that in classical fluid mechanics the relation between turbulence and fractals has been noticed for some time 77 81].

Finally we notice that there are aspects of what we have discussed which relate to classical fluid mechanics, particularly to the problem of the complexity of solutions of the Euler equation for an inviscid incompressible fluid. In the absence of viscous dissipation, classical vorticity can stretch greatly, and filamentary vortex structures may twist wildly [62, 82], unable to reconnect, and may even tend to a blow-up in finite time. But there are important differences between incompressible Euler vortices and superfluid vortices. Superfluid vortices reconnect, lose energy by radiating it away, and vortex volume is not conserved (the superfluid vortex core radius is fixed by quantum mechanical constraints on the rotation). Therefore Lagrangian methods for classical flows and for superfluid flows differ in both scope and detail. For example, Schwarz's de-singularization of the Biot-Savart integral does not involve a time-dependent core size. Another example is the cutoff $\delta$, which models sound dissipation and the reconnecting distance. In our case, dissipation and reconnections are essential ingredients of the problem, and the numerical method must model their existence. In the classical case, dissipation and reconnections are not part of the problem.

\section{ACKNOWLEDGEMENTS}

We thank W.F. Vinen, E. Kozik and V. L'vov for suggestions and discussions. We are greatly indebted to R. Hänninen, who stimulated the development of a better energy-conserving algorithm. 
[1] R.J. Donnelly, Quantized Vortices In Helium II, Cambridge University Press, Cambridge (1991).

[2] Quantized Vortex Dynamics And Superfluid Turbulence, Springer's Lecture Notes in Physics 571, edited by C.F. Barenghi, R.J. Donnelly and W.F. Vinen (2001).

[3] Vortices and Turbulence at Very Low Temperatures, edited by C.F. Barenghi and Y.A. Sergeev, CISM Courses and Lecture Notes, Springer (2008).

[4] P.-E. Roche, P. Diribarne, T. Didelot, O. Français, L. Rousseau and H. Willaime, Europhysics Lett. 77, 66002 (2007).

[5] M.R. Smith, R.J. Donnelly, N. Goldenfeld, and W.F. Vinen, Phys. Rev. Lett. 71, 2583 (1993).

[6] M. Blažková, D. Schmoranzer, L. Skrbek, and W.F. Vinen, Phys. Rev. B 79, 054522 (2009).

[7] R. Goto, S. Fujiyama, H. Yano, Y. Nago, N. Hashimoto, K. Obara, O. Ishikawa, M. Tsubota, and T. Hata, Phys. Rev. Lett. 100, 045301 (2008).

[8] W.F. Vinen, Proc. Roy. Soc. A240, 114 (1957).

[9] J.T. Tough, in Progress of Low Temperature Physics Vol. VIII, chapter 3, page 133, edited by D.F. Brewer (1982).

[10] M.S. Paoletti, R.B. Fiorito, K.R. Sreenivasan, and D.P. Lathrop, J. Phys. Soc. Japan. 77111007 (2008).

[11] C.F. Barenghi, A.V. Gordeev, and L. Skrbek, Phys. Rev. E 74, 026309 (2006).

[12] L. Skrbek, A.V. Gordeev, and F. Soukup, Phys. Rev. E 67, 047302 (2003).

[13] P.M. Walmsley and A.I. Golov, Phys. Rev. Lett. 100, 245301 (2008).

[14] V.B. Eltsov, A.I. Golov, R. de Graaf, R. Hänninen, M. Krusius, V.S. Lvov, and R.E. Solntsev, Phys. Rev. Lett. 99, 265301 (2007).

[15] D.I. Bradley, S.N. Fisher, A.M. Guénault, R.P. Haley, S. OSullivan, G.R. Pickett, and V. Tsepelin, Phys. Rev. Lett. 101, $065302(2008)$.

[16] E.A.L. Henn, J.A. Seman, G. Roati, K.M.F. Magalhães, and V.S. Bagnato, Phys. Rev. Lett. 103, 045301 (2009).

[17] A.C. White, C.F. Barenghi, N.P. Proukakis, A.J. Youd, and D.H. Wacks, Phys. Rev. Lett. 104, 075301 (2010).

[18] C.F. Barenghi, W.F. Vinen and R.J. Donnelly, J. Low Temp. Physics 52, 189-247 (1982).

[19] S.I. Davis, P.C. Hendry and P.V.E. McClintock, Physica B 280, 43 (2000).

[20] D.I. Bradley, D.O. Clubb, S.N. Fisher, A.M. Guenault, R.P. Haley, C.J. Matthews, G.R. Pickett, V. Tsepelin and K. Zaki, Phys. Rev. Lett. 96, 035301 (2006).

[21] B.V. Svistunov, Phys. Rev. B 52, 3647 (1995).

[22] D. Kivotides, J.C. Vassilicos, D.C. Samuels and C.F. Barenghi, Phys. Rev. Letters, 86, 3080 (2001).

[23] W.F. Vinen, M. Tsubota and A. Mitani, Phys. Rev. Lett. 91, 135301 (2003).

[24] W. Thomson, Phil. Mag. 10, 155 (1880).

[25] J.J. Thomson, A Treatise on the Motion of Vortex Rings, Macmillan, London (1883).

[26] H.C. Pocklington, Philos. Trans. R. Soc. London Ser. A 186603 (1895).

[27] C.F. Barenghi, R.J. Donnelly and W.F. Vinen, Phys. Fluids 28, 498 (1985).

[28] P.H. Roberts, Proc. Roy. Soc. London A 459, 597 (2003).

[29] L.P. Pitaevskii, Sov. Phys. JETP 13, 451 (2003)

[30] W.F. Vinen, Phys. Rev. B 64134520 (2001).

[31] M. Leadbeater, D.C. Samuels, C.F. Barenghi and C.S. Adams, Phys. Rev. A 67, 015601 (2003).

[32] C.F. Barenghi, N.G. Parker, N.P. Proukakis and C.S. Adams, J. Low Temp. Phys. 138, 629 (2005).

[33] E. Kozik and B. Svistunov, Phys. Rev. B 72, 172505 (2005).

[34] U. Frisch, Turbulence, Cambridge University Press (1995).

[35] P.A. Davidson, Turbulence, Oxford University Press (2004).

[36] W.F. Vinen and J.J. Niemela, J. Low Temp. Phys. 128, 167 (2002) and Erratum, 129 , 213 (2002).

[37] S. Goto, J. Fluid Mech. 605, 355 (2008).

[38] C.F. Barenghi, S. Hulton and D.C. Samuels, Phys. Rev. Letters 89275301 (2002).

[39] V.S. L'vov, S.V. Nazarenko and O. Rudenko, Phys. Rev. B 76024520 (2007).

[40] V.S. L'vov, S.V. Nazarenko and O. Rudenko, J. Low Temp. Phys. 153140 (2008).

[41] J. Maurer and P. Tabeling, Europhys. Lett. 43, 29 (1998)

[42] T. Araki, M. Tsubota and S.K. Nemirovskii, Phys. Rev. Lett. 89, 145301 (2002).

[43] C. Nore, M. Abid, and M.E. Brachet, Phys. Rev. Lett. 78, 3896 (1997).

[44] M. Kobayashi and M. Tsubota, Phys. Rev. Lett. 94, 065302 (2005).

[45] J. Yepez, G. Vahala, L. Vahala and M. Soe, Phys. Rev. Lett. 103084501 (2009).

[46] V.S. L'vov, S.V. Nazarenko, G.E. Volovik, JETP Lett. 80, 479 (2004).

[47] V.S. L'vov, S.V. Nazarenko and L. Skrbek, J. Low Temp. Physics 145, 125 (2006).

[48] S.V. Nazarenko, JETP Lett. 83, 198 (2006).

[49] D. Proment, S.V. Nazarenko and M. Onorato, Phys. Rev. A 80 051603(R) (2009).

[50] G. Boffetta, A. Celani, D. Dezzani, J. Laurie and S.V. Nazarenko J. Low Temp. Phys 156, 193 (2009).

[51] V.S. L'vov and S.V. Nazarenko, JETP Letters 91, 464 (2010).

[52] E. Kozik and B. Svistunov, Phys. Rev. Lett. 92, 035301 (2004).

[53] E. Kozik and B. Svistunov, Phys. Rev. Lett. 94, 025301 (2005).

[54] E. Kozik and B. Svistunov, Phys. Rev. Lett. 100, 195302 (2008).

[55] E. Kozik and B. Svistunov, Phys. Rev. B 77, 060502(R) (2008). 
[56] E. Kozik and B. Svistunov, J. Low Temp. Phys. 156, 215 (2009).

[57] T. V. Chagovets, A. V. Gordeev, and L. Skrbek, Phys. Rev. E 76, 027301 (2007).

[58] P. M. Walmsley, A. I. Golov, H. E. Hall, A. A. Levchenko, and W. F. Vinen, Phys. Rev. Lett. 99, 265302 (2007).

[59] K.W. Schwarz, Phys. Rev. B 38, 2398 (1988).

[60] K.W. Schwarz, Phys. Rev. B 31, 5782 (1985)

[61] A. Leonard, Ann. Rev. Fluid Mech. 17, 523 (1985).

[62] E.D. Siggia, Phys. of Fluids, 28, 794 (1985).

[63] L. Gamet, F. Ducros, F. Nicoud,and T. Poinsot, International Journal for Numerical Methods in Fluids 29, 2 (1999)

[64] J. Koplik and H. Levine, Phys. Rev. Lett. 71, 1375 (1993); R. Tebbs, A.J. Youd and C.F. Barenghi, J. Low Temp. Physics, 162, $314(2010)$.

[65] M. Leadbeater, T. Winiecki, D.C. Samuels, C.F. Barenghi and C.S. Adams, Phys. Rev. Lett. 86, 1410 (2001).

[66] P.G. Saffman, Vortex Dynamics, Cambridge University Press (1992).

[67] J.P. Laurie, Six-wave systems in one dimensional wave turbulence, PhD thesis, University of Warwick, 2010.

[68] S.Z. Alamri, A.J. Youd and C.F. Barenghi, Phys. Rev. Lett. 101, 215302 (2008)

[69] R.M. Kerr, arXiv:1006.3911v1, 20 June 2010.

[70] S.V. Nazarenko and A.A. Shekochihin, arXiv:0904.3488v2 (28 Dec 2009).

[71] P. Grassberger and I.Procaccia, Physica D 9, 189 (1983).

[72] M. Tsubota, T. Araki and S.K. Nemirovskii, Phys. Rev. B 6211751 (2000).

[73] D. Kivotides, C.F. Barenghi and D.C. Samuels, Phys. Rev. Lett. 87, 155301 (2001).

[74] F. Maggioni, S.Z. Alamri, C.F. Barenghi and R.L. Ricca, Il Nuovo Cimento C 32, 133 (2009).

[75] F. Maggioni, S. Alamri, C.F. Barenghi and R.L. Ricca, Phys. Rev. E 82026309 (2010).

[76] D. Jou, M.S. Mongiovi', M. Sciacca and C.F. Barenghi, J. Physics A: Math. Theor. 43205501 (2010).

[77] B.B. Mandelbrot J. Fluid Mech. 72, 401 (1975).

[78] K.R. Sreenivasan, Ann. Rev. Fluid Mech. 23539 (1991).

[79] J.C.H. Fung and J.C. Vassilicos, Phys. Fluids A 32725 (1991).

[80] I. Procaccia, A. Brandenburg, M.H. Jensen, and A. Vincent, Europhys. Lett. 19183 (1992).

[81] P.L. Vanyan, JETP Lett. 60, 258 (1994).

[82] A.J. Chorin, Commun. Math. Phys. 83, 517 (1982). 


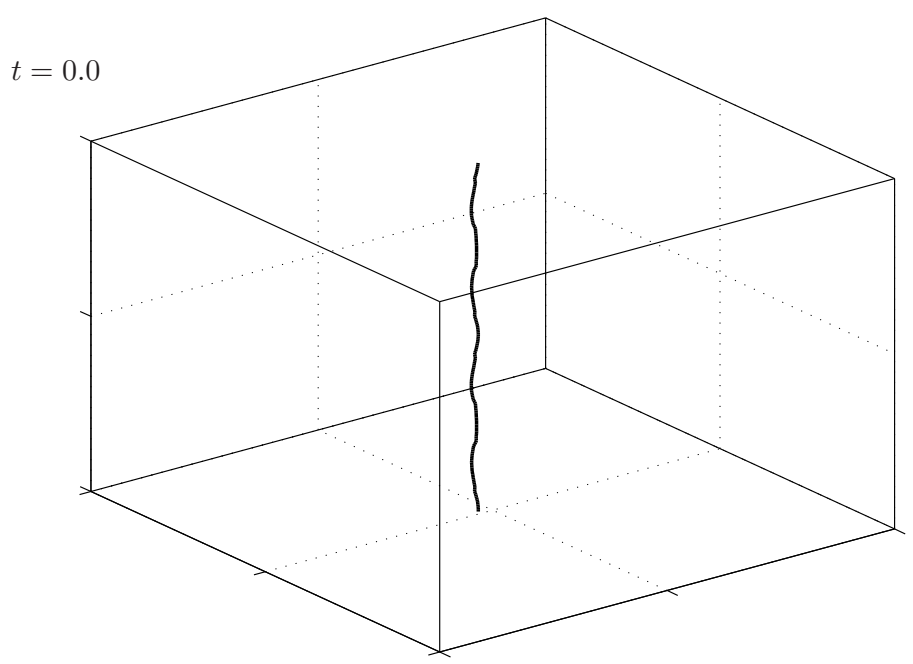

FIG. 1: Single vortex line initially perturbed by two Kelvin waves of wavenumbers $k=3$ and $k=4$ (where $k=2 \pi / \lambda)$ and amplitudes $A=3 \delta$ and $A=1.2 \delta$, where $\delta=0.001 \mathrm{~cm}$. The vortex line is contained in a cubic periodic box of size $D=0.1 \mathrm{~cm}$. This initial condition does not evolve into a Kelvin-waves cascade. 

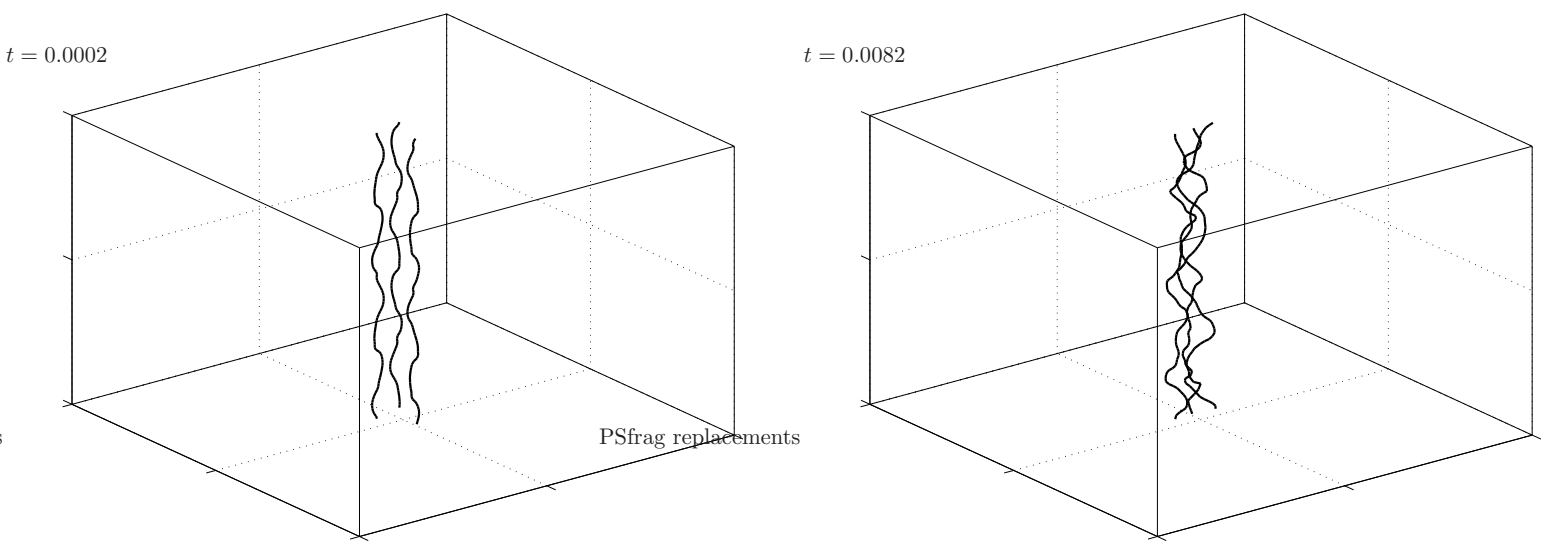

FIG. 2: Time evolution of three parallel vortex lines in a cubic periodic box of size $D=0.1 \mathrm{~cm}$. Initially, at $t=0$ (left), each vortex line is perturbed by two Kelvin waves of wavenumbers $k=3$ and $k=4$ and amplitudes $A=3 \delta$ and $A=1.2 \delta$, as in Fig. 1 The waves are offset by random phases. This initial condition triggers a Kelvin-waves cascade, as shown by the increasing wiggliness at time $t=0.0082 \mathrm{~s}$ (right). 


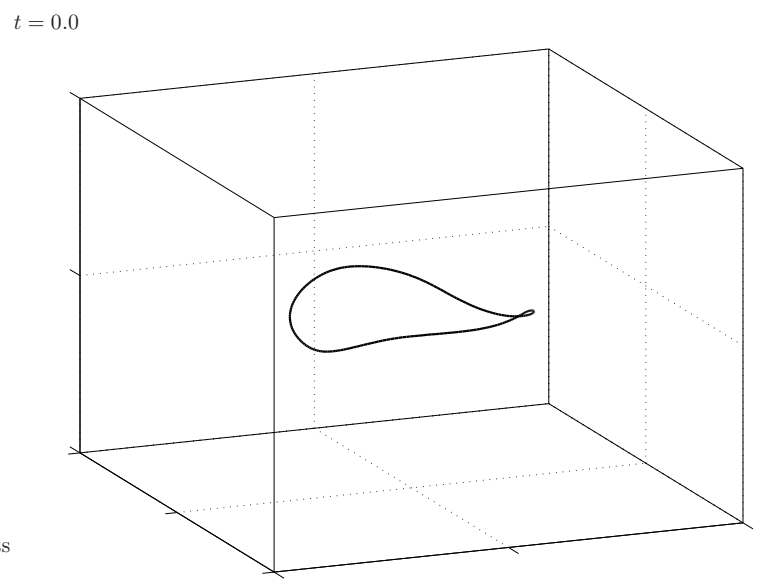

FIG. 3: Single vortex ring of radius $R=0.1 \mathrm{~cm}$ perturbed by two Kelvin waves of mode numbers $m=2$ and $m=5$ (where $\lambda=2 \pi R / m$ ) and amplitudes $A=5 \delta$ and $A=1.4 \delta$, where $\delta=0.001 \mathrm{~cm}$. The vortex ring is contained in an infinite volume and the box (of size $D=0.1 \mathrm{~cm}$ ) is for visualization only. This initial condition does not evolve into a Kelvin-waves cascade. 

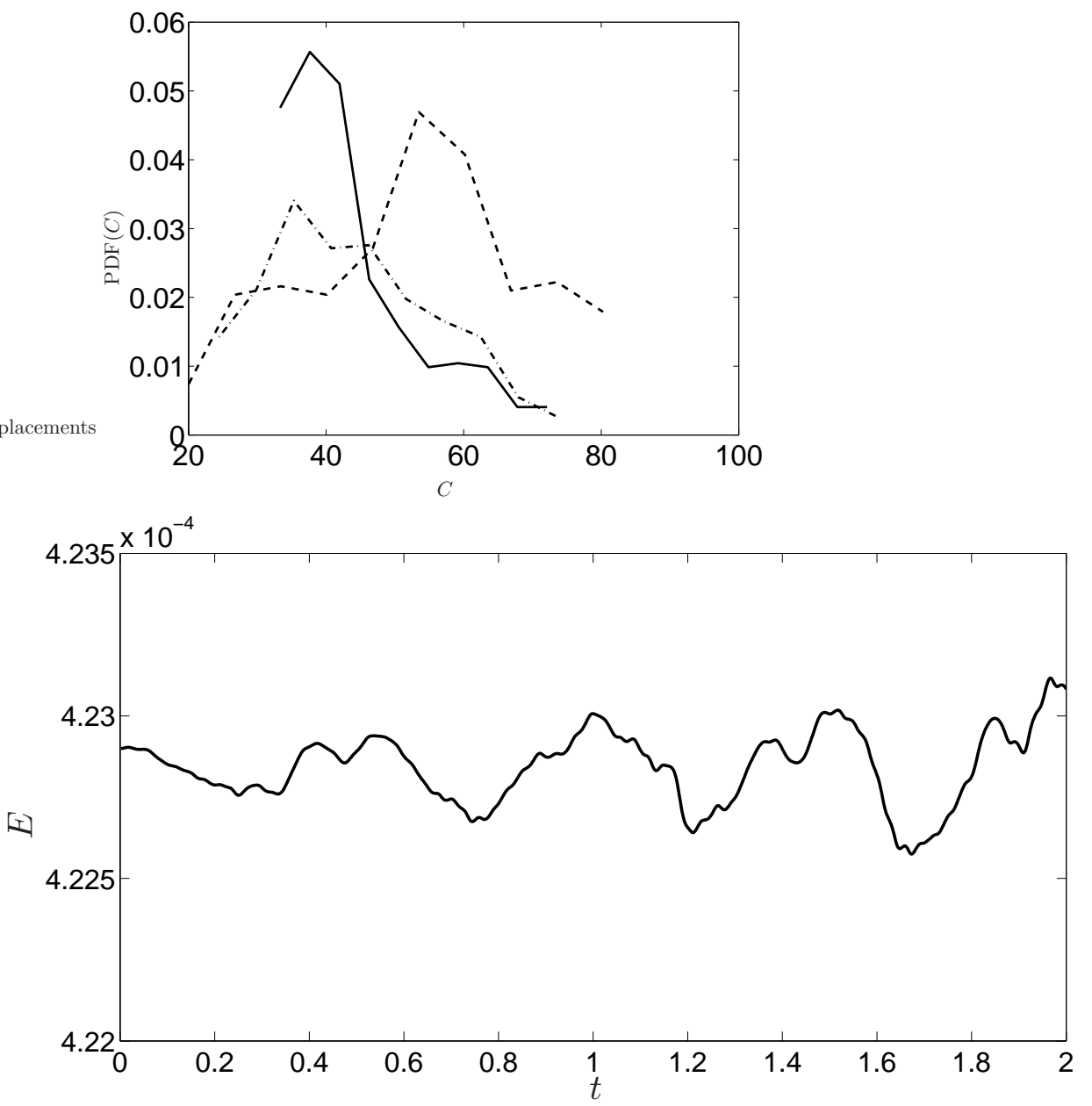

FIG. 4: Evolution of curvature and energy corresponding to the non-cascading initial condition shown in Fig. 3 Top: PDF $(C)$ vs $C\left(\mathrm{~cm}^{-1}\right)$ at different times. Solid line: at $t=0$; dashed line: at $t=0.5 \mathrm{~s}$; dot-dashed line: at $t=1 \mathrm{~s}$. Note that the peak of the PDF does not move significantly to larger values of $C$ with time $t$. Bottom: Energy $E\left(\mathrm{~cm}^{2} / \mathrm{s}^{2}\right)$ vs time $t(\mathrm{~s})$. 

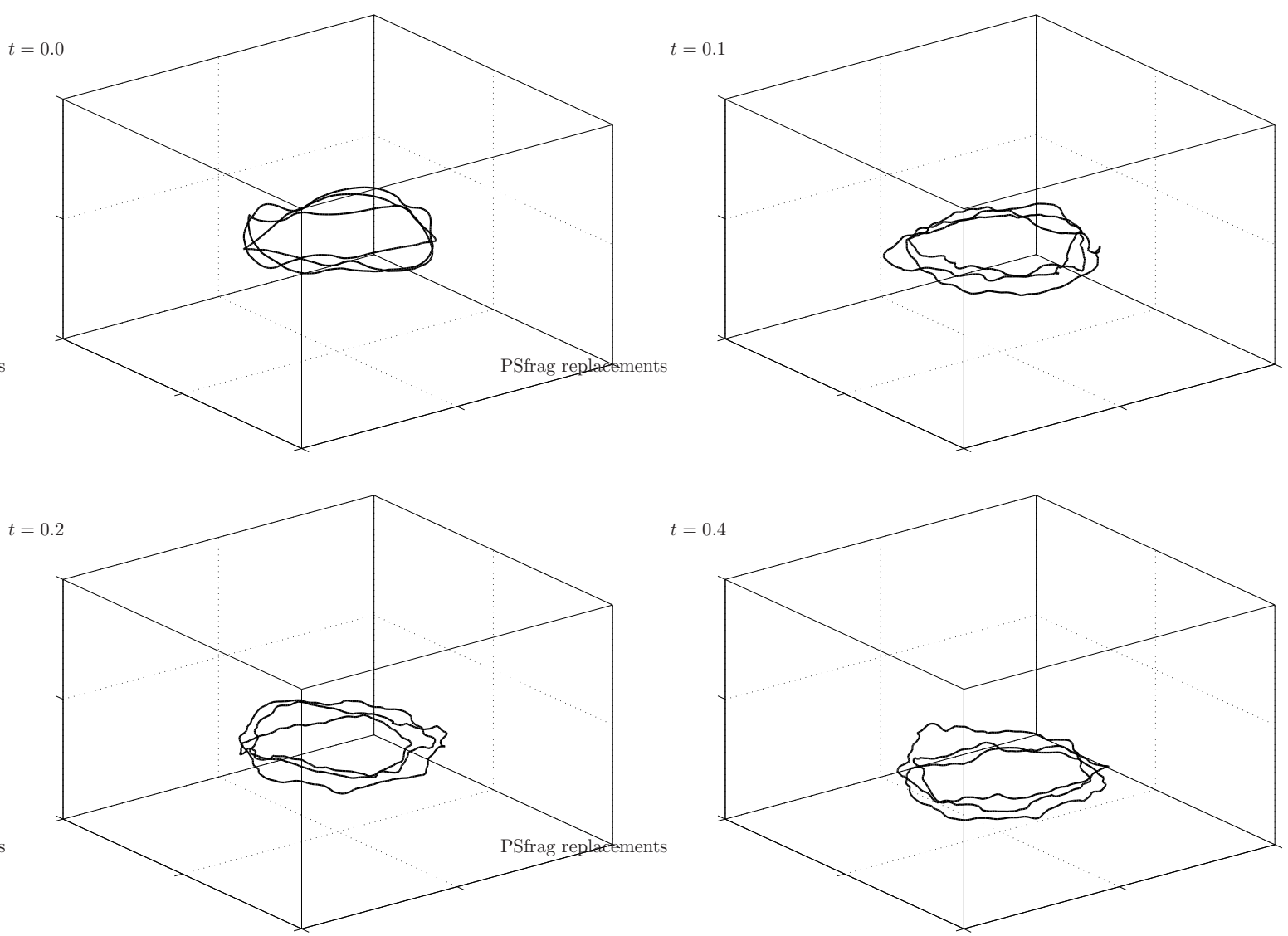

FIG. 5: Cascading vortex configuration. Time evolution of three vortex rings of radii $R_{1}=R_{2}=R_{3}=0.16 \mathrm{~cm}$. Each ring has been perturbed by two Kelvin waves. Two rings have waves with mode numbers $m=3$ and $m=4$ and amplitudes $A=5 \delta$ and $A=1.4 \delta$; one ring has waves with $m=2$ and $m=3$ and $A=5 \delta$ and $A=1.4 \delta$. As in Fig. 2, this initial condition triggers a Kelvin-waves cascade (note the increasing wiggliness of the filaments). The calculation is performed in an infinite volume: the box shown (for visualisation purpose) is a cube with sides of length $0.1 \mathrm{~cm}$. Top left: at time $t=0 \mathrm{~s}$; Top right: at $t=0.1 \mathrm{~s}$; Bottom left: at $t=0.2 \mathrm{~s}$; Bottom right: at $t=0.4 \mathrm{~s}$. 


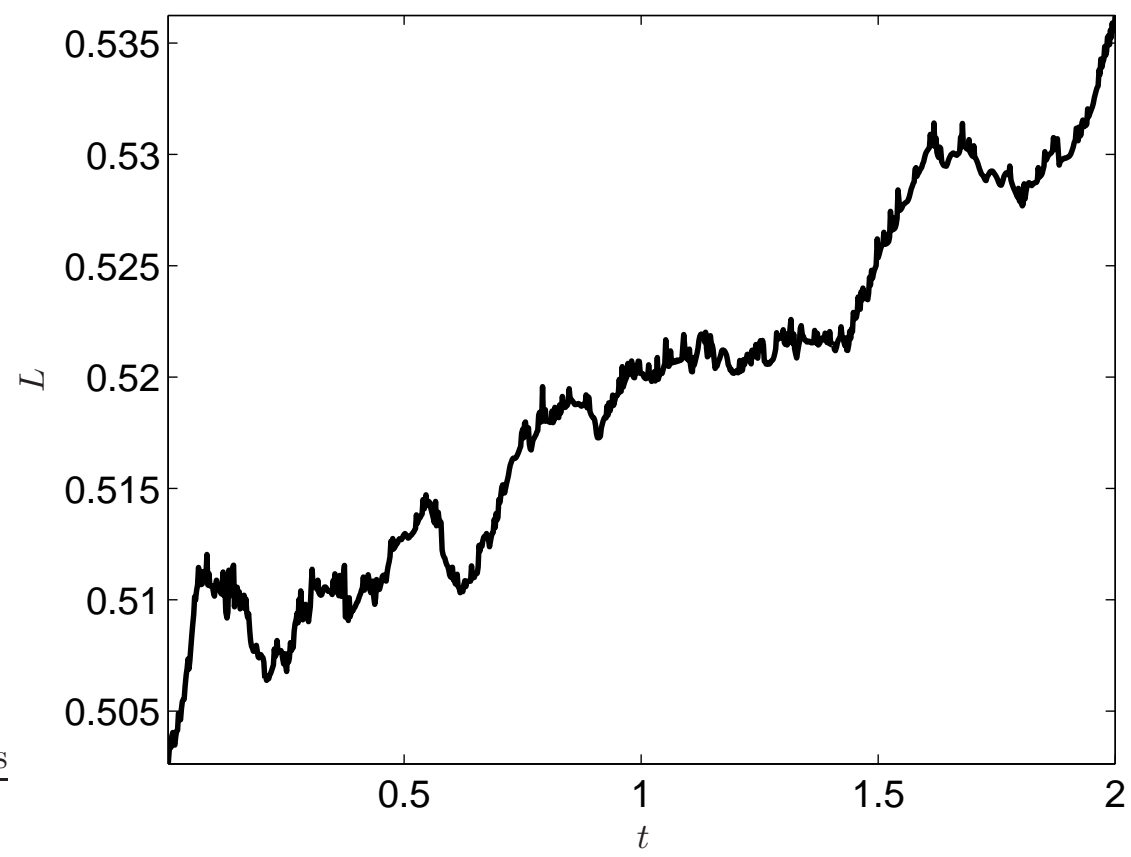

FIG. 6: Vortex length $L(\mathrm{~cm})$ vs time $t(\mathrm{~s})$ corresponding to Fig. 5 


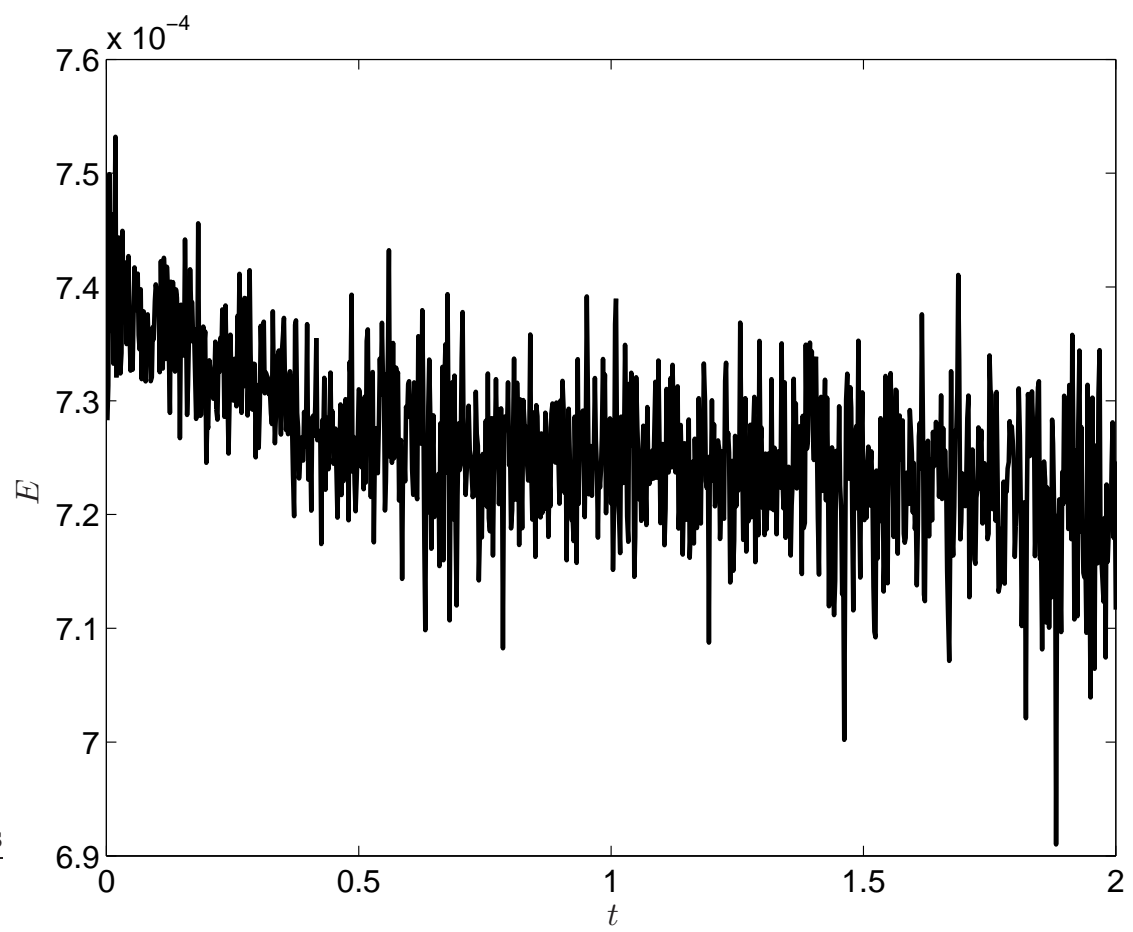

FIG. 7: Energy $E\left(\mathrm{~cm}^{2} / \mathrm{s}^{2}\right)$ vs time $t(\mathrm{~s})$ corresponding to Fig. 5 . 

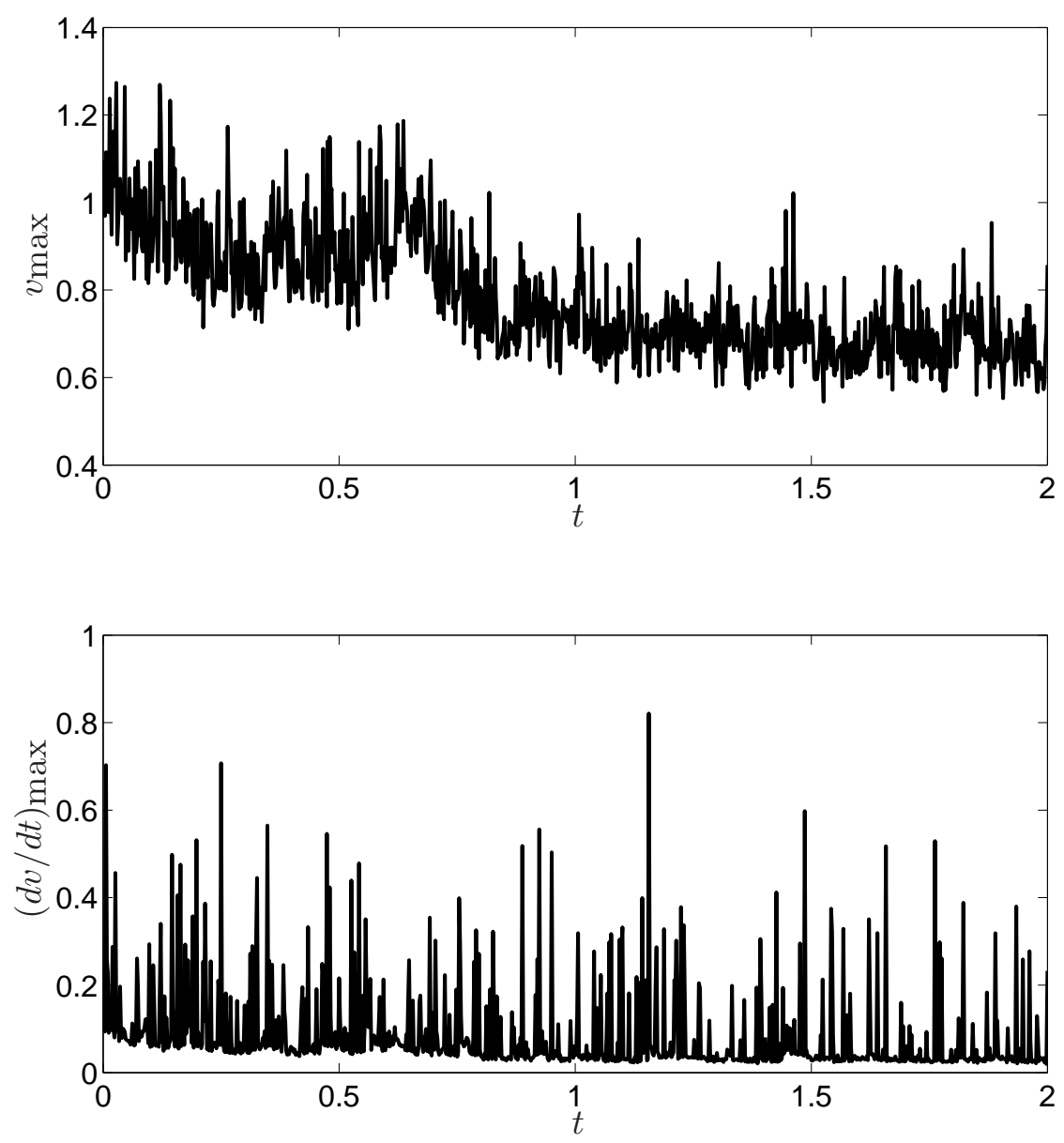

FIG. 8: Top: maximum speed $v_{\max }(\mathrm{cm} / \mathrm{s})$ vs time $t(\mathrm{~s})($ top $)$ and maximum acceleration $(d v / d t)_{\max }\left(\mathrm{cm} / \mathrm{s}^{2}\right)$ vs $t(\mathrm{~s})(\mathrm{bottom})$ of the vortex points $\mathbf{s}_{j}(j=1, \cdots N)$ corresponding to Fig. 5 . 

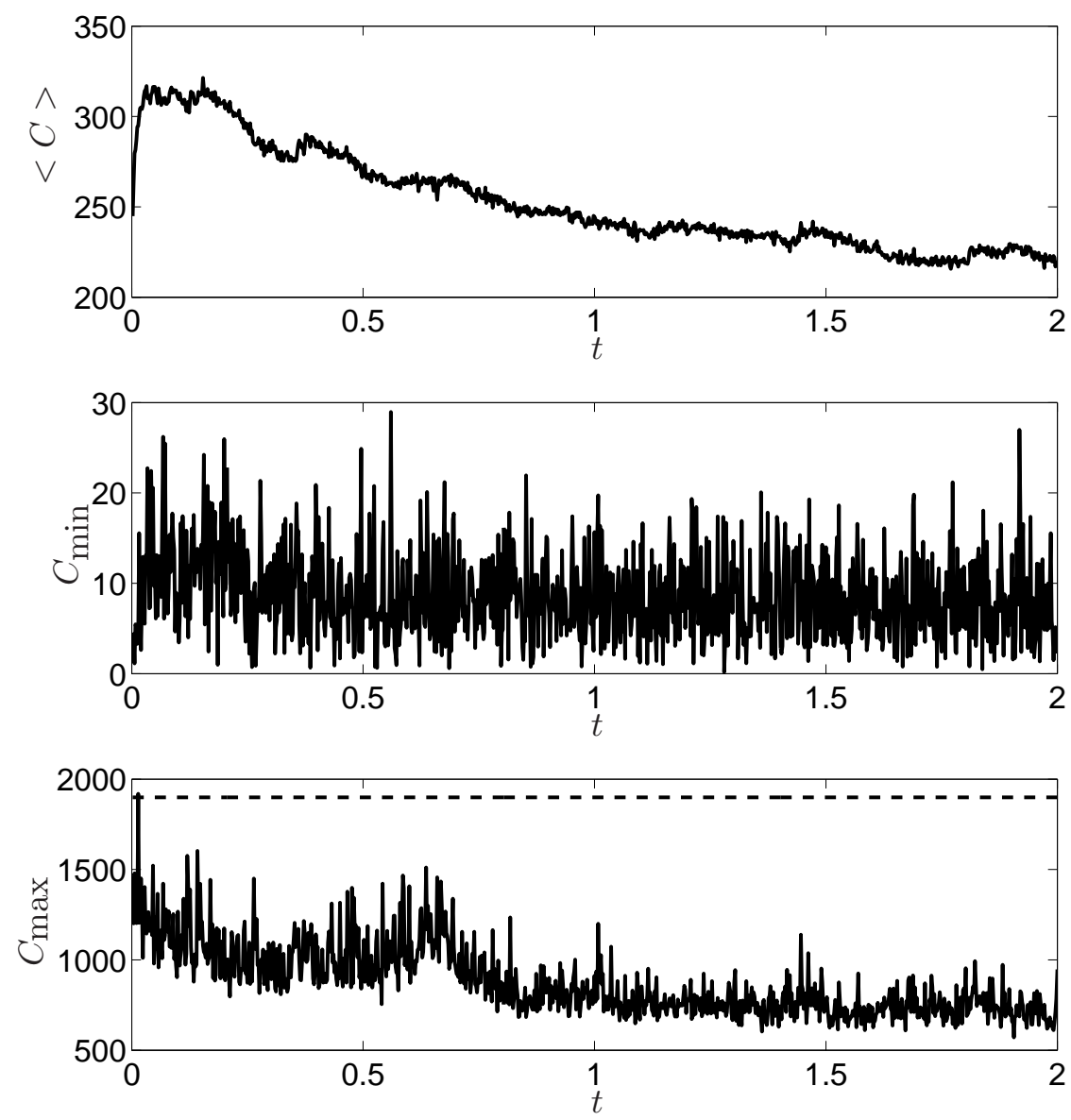

FIG. 9: Curvature vs time $t$ corresponding to Fig. 5. Top: mean curvature $\langle C\rangle\left(\mathrm{cm}^{-1}\right)$ vs $t$ (s). Middle: minimum curvature $C_{\min }\left(\mathrm{cm}^{-1}\right)$ vs $t(\mathrm{~s})$. Bottom: maximum curvature $C_{\max }\left(\mathrm{cm}^{-1}\right)$ vs $t(\mathrm{~s})$; the dashed line is the maximum curvature which corresponds to the numerical discretization. The maximum curvature which is observed is therefore always much less than the numerical resolution. 


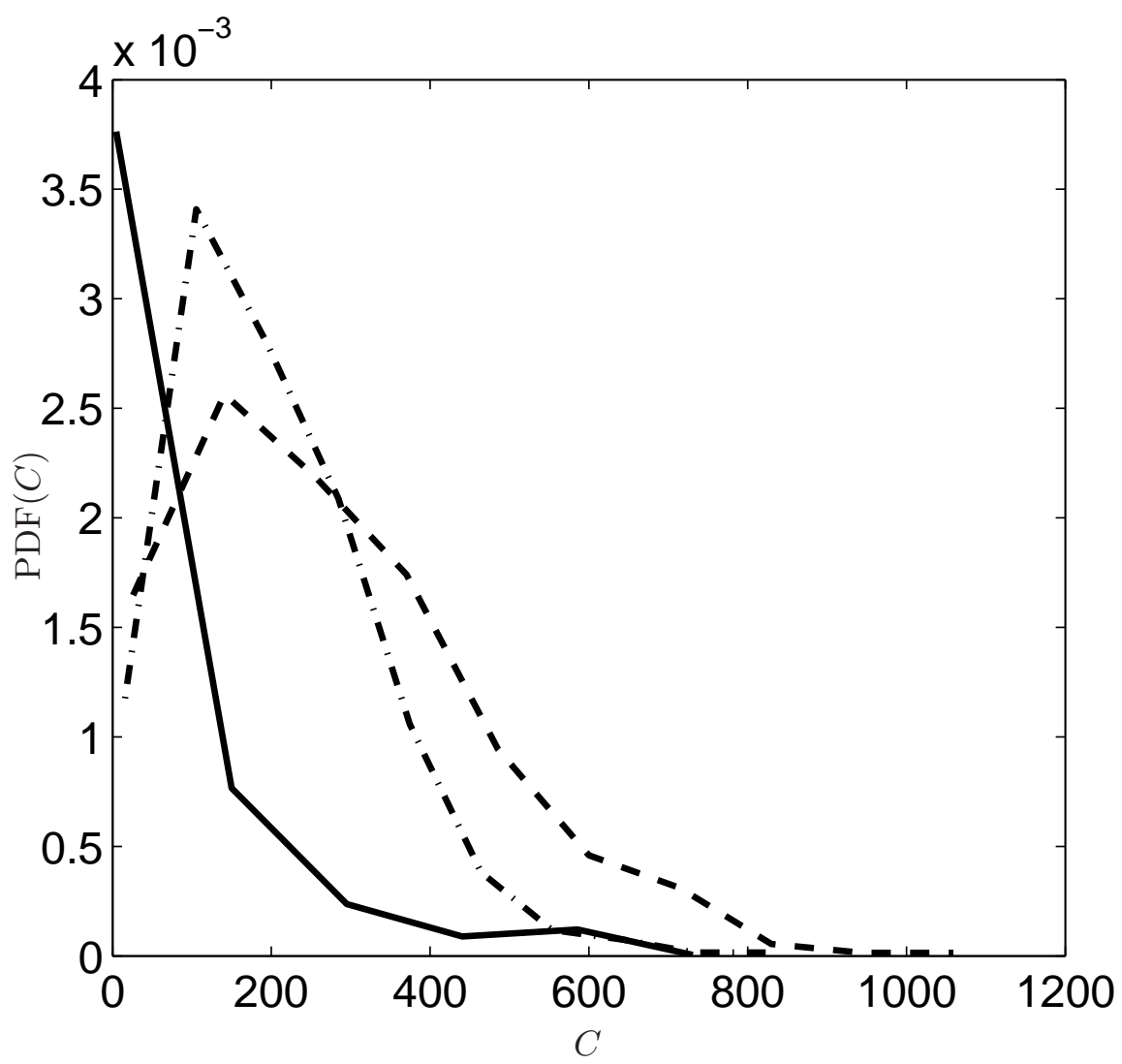

FIG. 10: Probability density function of the curvature PDF(C) vs $C\left(\mathrm{~cm}^{-1}\right)$ corresponding to Fig. 5 at selected times. Solid line: at $t=0$. Dashed line: at $t=0.5 \mathrm{~s}$. Dot-dashed line: at $t=1 \mathrm{~s}$. Note the curve initially moves greatly to the right (compared to the non-cascading vortices shown in Fig. 4(top)) and then, at later times, slightly to the left (hence lower curvature) as the turbulence slowly decays. 


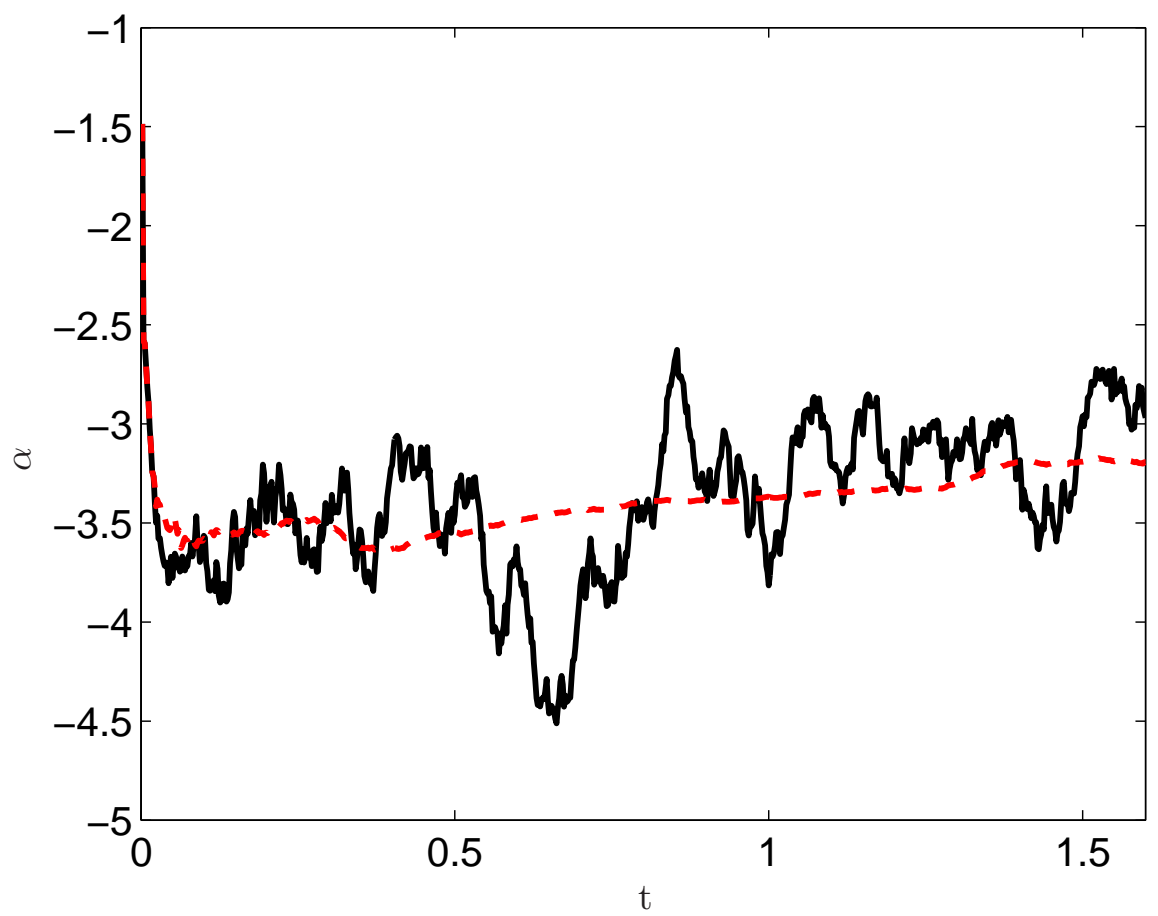

FIG. 11: (Color online). Exponent $\alpha$ vs time $t$ of the probability density function of the curvature, $\mathrm{PDF}(\mathrm{C}) \sim C^{\alpha}$ for large $C$, corresponding to Fig. [5 The (red) dashed line is the running average. 

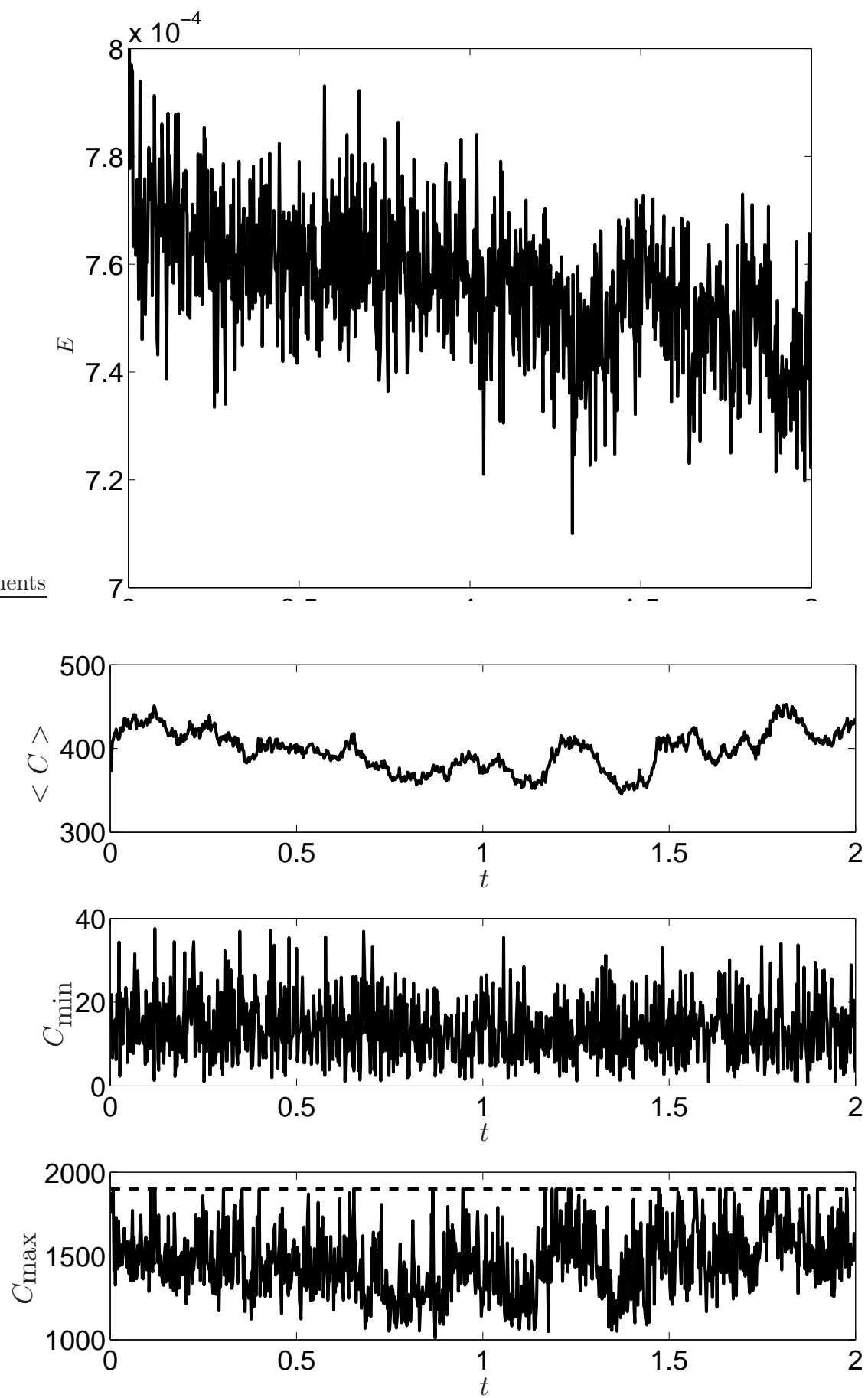

FIG. 12: Analysis of the evolution of the initial vortex configuration shown in Fig. 5 (top left) in the absence of vortex reconnections. Top: Energy $E\left(\mathrm{~cm}^{2} / \mathrm{s}^{2}\right)$ vs time $t(\mathrm{~s})$. Bottom: Corresponding mean curvature $\langle C\rangle$ vs $t$, miniumum curvature $C_{\min }$ vs $t$, and maximum curvature $C_{\max }$ vs $t$. As in Fig. 9(bottom), the dashed line is the maximum curvature which corresponds to the numerical discretization. 

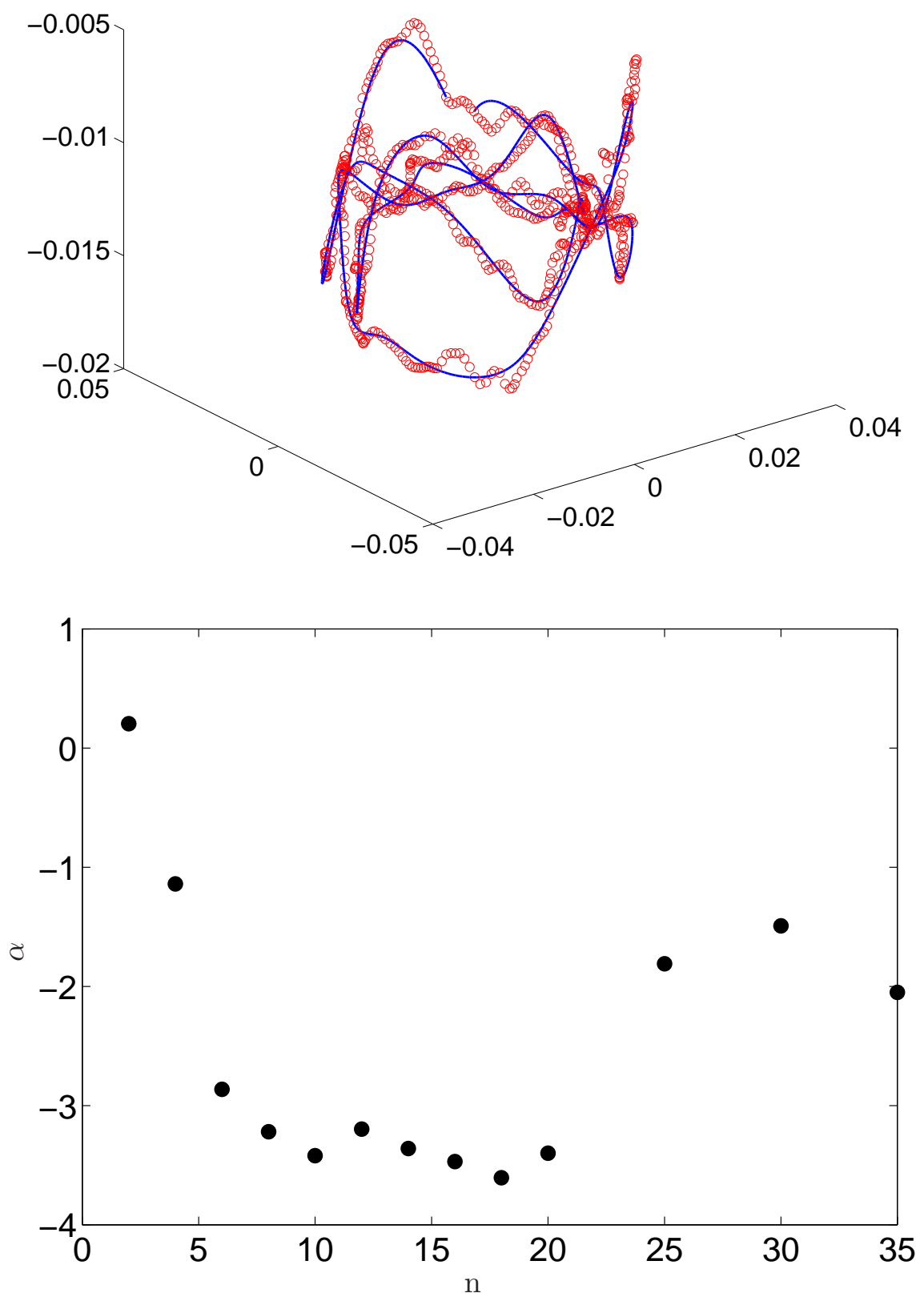

FIG. 13: (Color online). Top: Smoothed vortex configuration. The (red) circles denote the actual discretization points $\mathbf{s}_{j}$ $(j=1, \cdots N)$ of the vortex configuration corresponding to Fig. 5 at time $t=0.18 \mathrm{~s}$. The (blue) line is the smoothed curve curve $\mathbf{s}_{\text {smooth }}$ obtained by cubic spline fit interpolation over every $n^{\text {th }}=15$ points. Bottom: Slope $\beta$ of the amplitude spectrum $A(k) \sim k^{\beta}$ for large $k$ plotted as a function of $n$. Note the plateau $10<n<20$ where the spectral slope is roughly constant. 


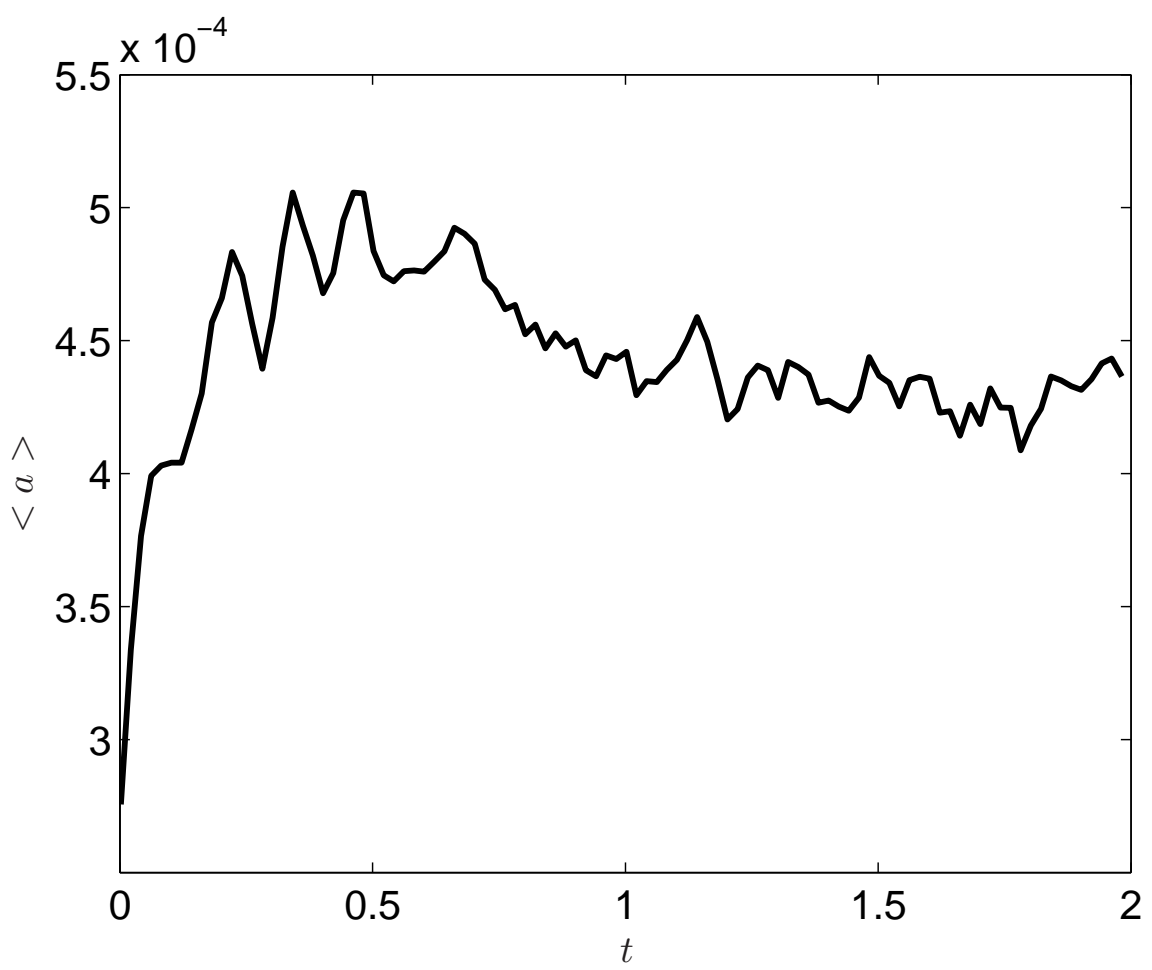

FIG. 14: Mean amplitude $\langle a\rangle(\mathrm{cm})$ of the Kelvin waves vs time $t \mathrm{~s}$ corresponding to Fig. 13 Note the saturation for $t>1(\mathrm{~s})$. 


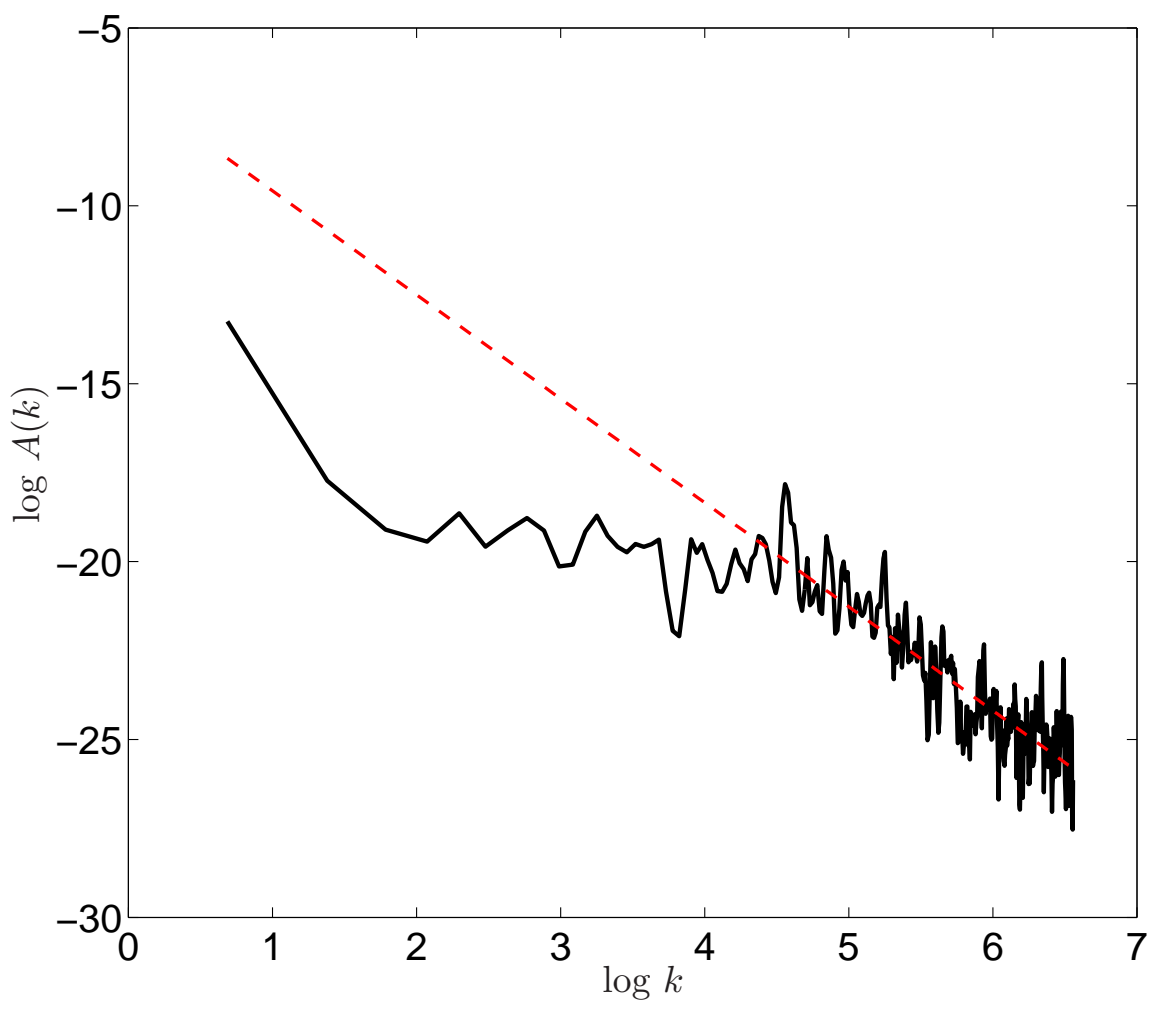

FIG. 15: (Color online) Log log plot of Kelvin wave amplitude spectrum $A(k)\left(\mathrm{cm}^{4}\right)$ vs wavenumber $k\left(\mathrm{~cm}^{-1}\right)$ at time $t=0.18$ (s), corresponding the Fig. 13. The (red) dashed line represents the large-k behaviour $A(k) \sim k^{\beta}$ with the best fit $\beta=-3.1$ 


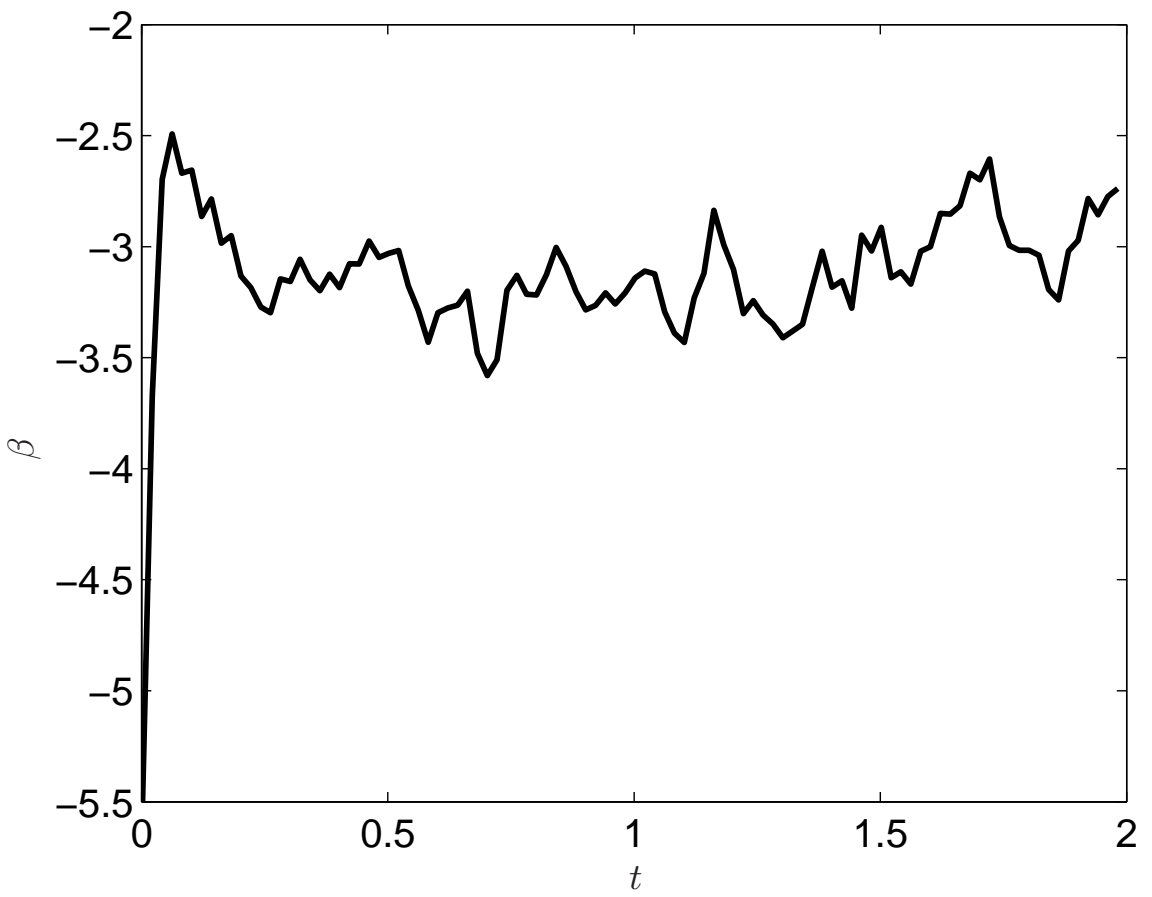

FIG. 16: Slope $\beta$ of the large-k amplitude spectrum $A(k) \sim k^{\beta}$ vs time $t$ s. Note the quick saturation. 

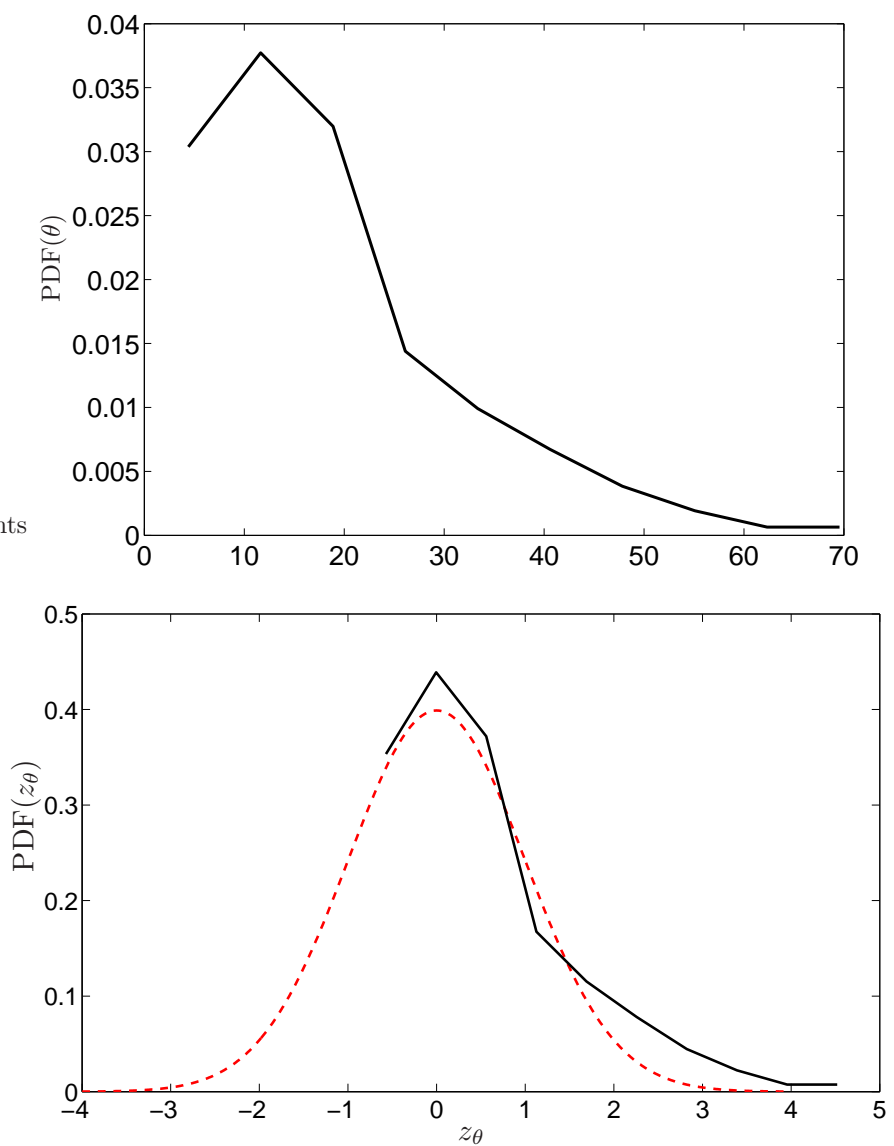

FIG. 17: (Color online). Angle between vortex filament and smoothed vortex filament. Top: Probability density function $\operatorname{PDF}(\theta)$ vs $\theta$ of the angle $\theta$ (in degrees) between the smoothed vortex filament and the actual vortex filament at $t=0.2 \mathrm{~s}$. Bottom: Probability density function $\operatorname{PDF}\left(z_{\theta}\right)$ vs $z_{\theta}$ of the scaled angle $z_{\theta}=(\theta-\bar{\theta}) / \sigma(\theta)$ where $\bar{\theta}$ and $\sigma(\theta)$ are respectively the mean and the variance of the distribution of the angle $\theta$. The dashed (red) line shows the normal PDF. Note the slight departure from Gaussianity at large angles. 

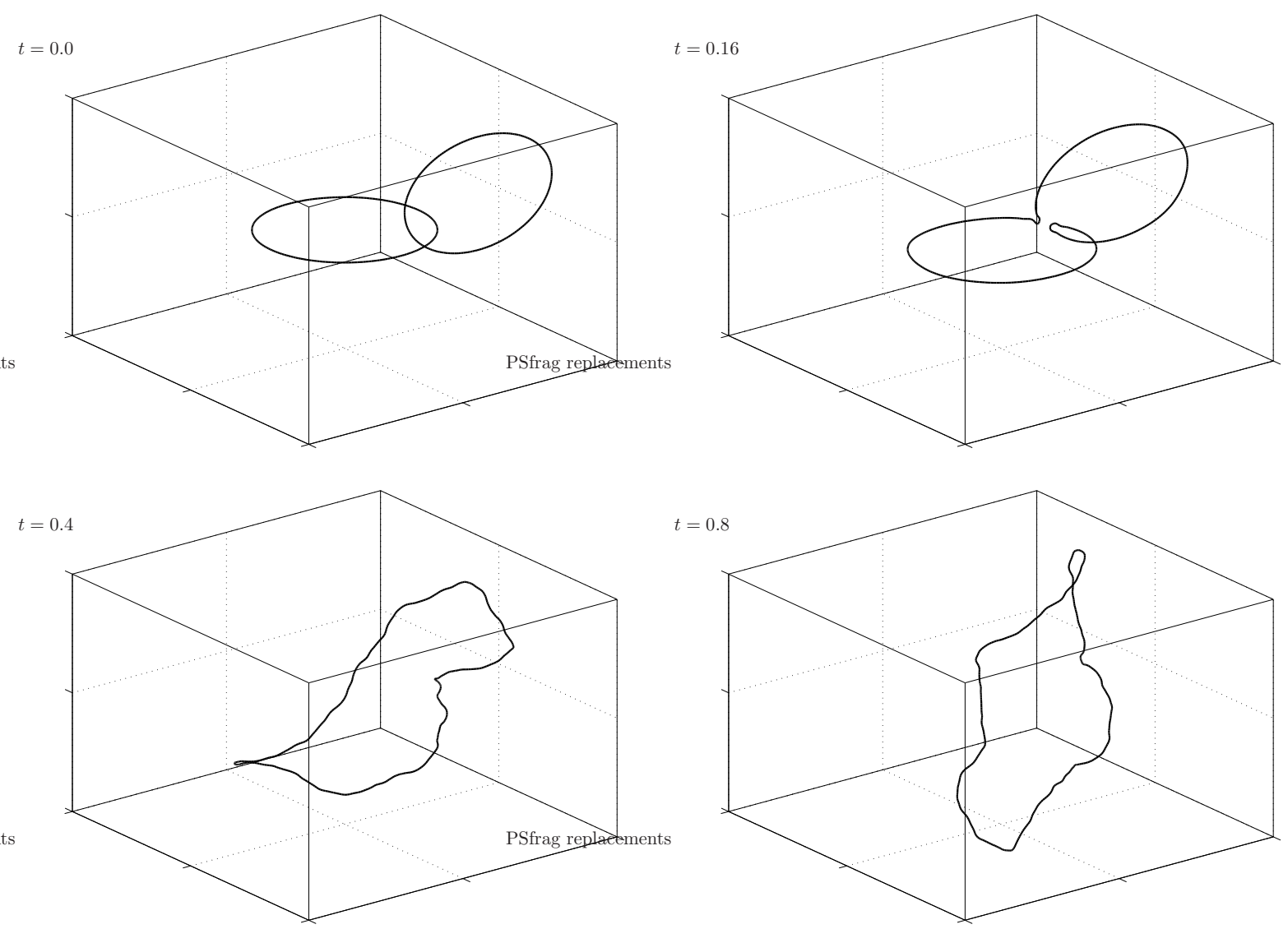

FIG. 18: Non-cascading reconnecting vortex rings. Time evolution of two rings of radius $R=0.16 \mathrm{~cm}$ initially linked to each other. Note that the reconnection cusp relaxes and induces Kelvin waves, but there is no cascade. Top left: At time $t=0 \mathrm{~s}$; Top right: $t=0.16 \mathrm{~s}$; Bottom left: $t=0.5 \mathrm{~s}$; Bottom right: $t=0.8 \mathrm{~s}$. 

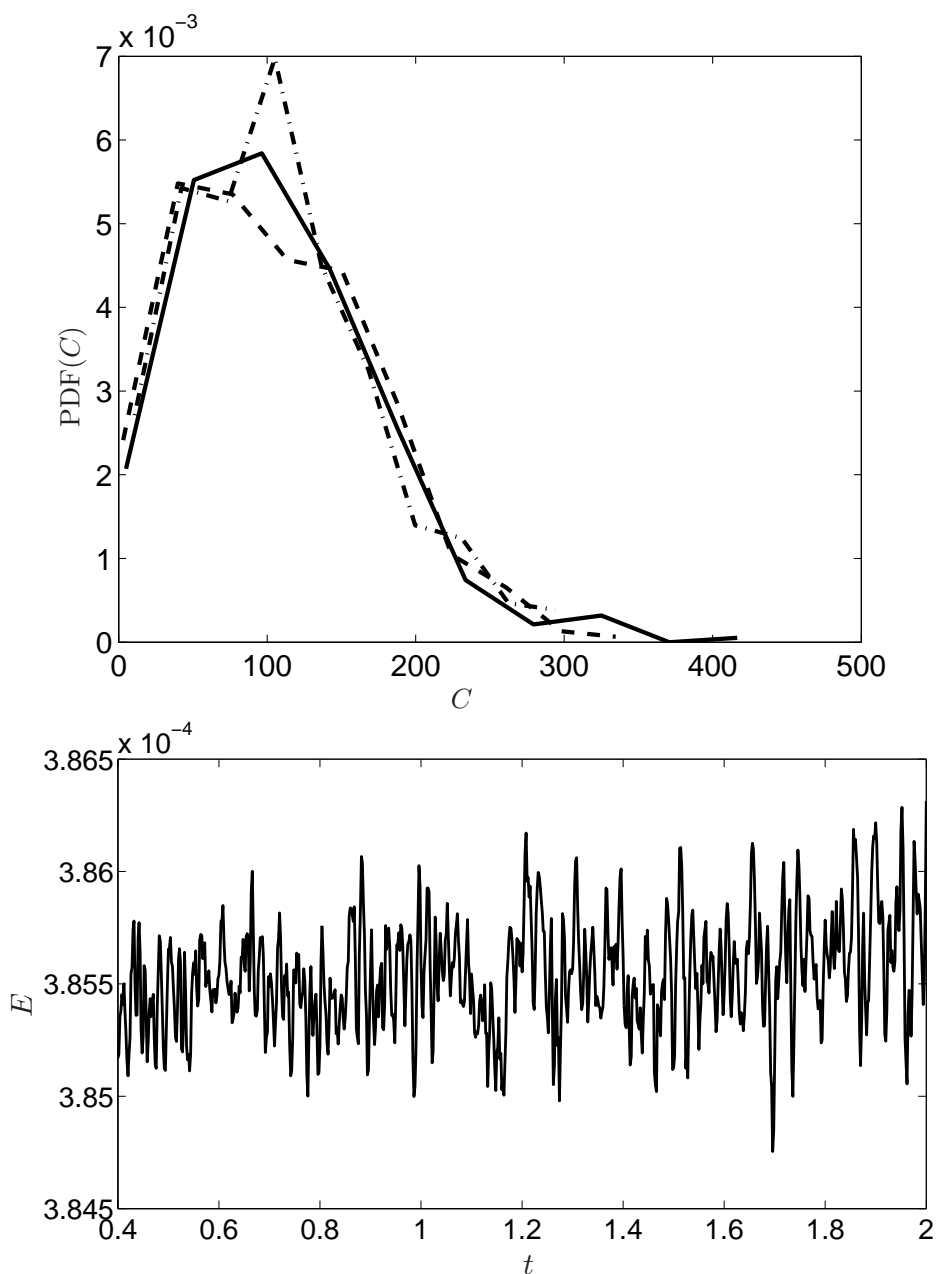

FIG. 19: Evolution of curvature and energy corresponding to Fig. 18 Top: $\operatorname{PDF}(C)$ vs $C\left(\mathrm{~cm}^{-1}\right)$ at $t=0$ (solid line), $t=0.2 \mathrm{~s}$ (dashed line) and $t=1.6 \mathrm{~s}$ (dot dashed line) Note that the peak of the PDF does not move to larger values of $C$. Bottom: Corresponding behaviour of the energy $E\left(\mathrm{~cm}^{2} / \mathrm{s}^{2}\right)$ vs time $t(\mathrm{~s})$. 

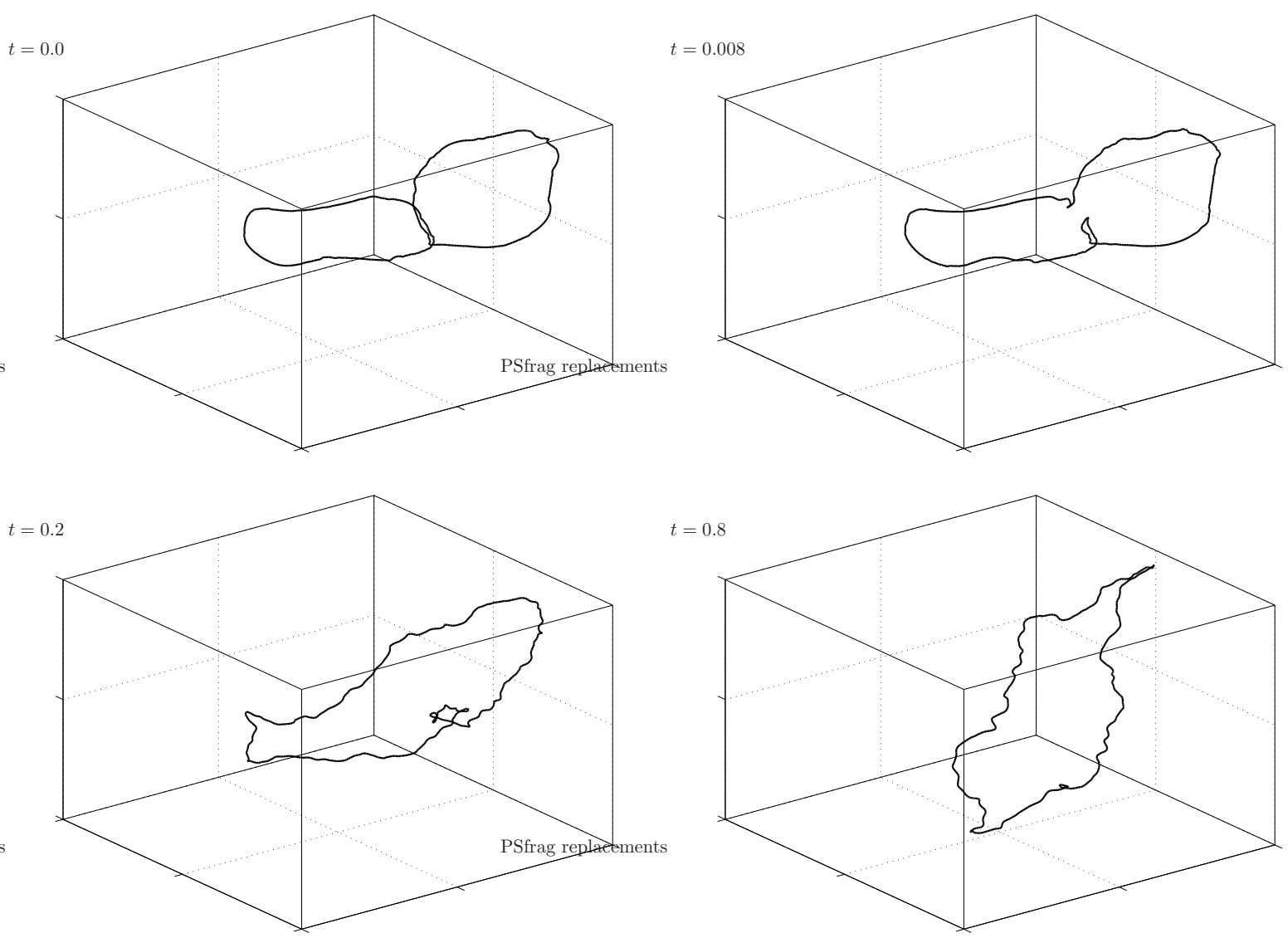

FIG. 20: Cascading reconnecting vortex rings. Time evolution of two rings of radius $R=0.16 \mathrm{~cm}$ initially linked to each other. This initial configuration differs from the configuration of Fig. (15) only because some Kelvin waves have been superimposed to the vortex rings. Note the development of the Kelvin waves cascade. Top left: At time $t=\mathrm{s}$; Top right: $t=\mathrm{s}$; Bottom left: $t=\mathrm{s}$; Bottom right: $t=\mathrm{s}$. 

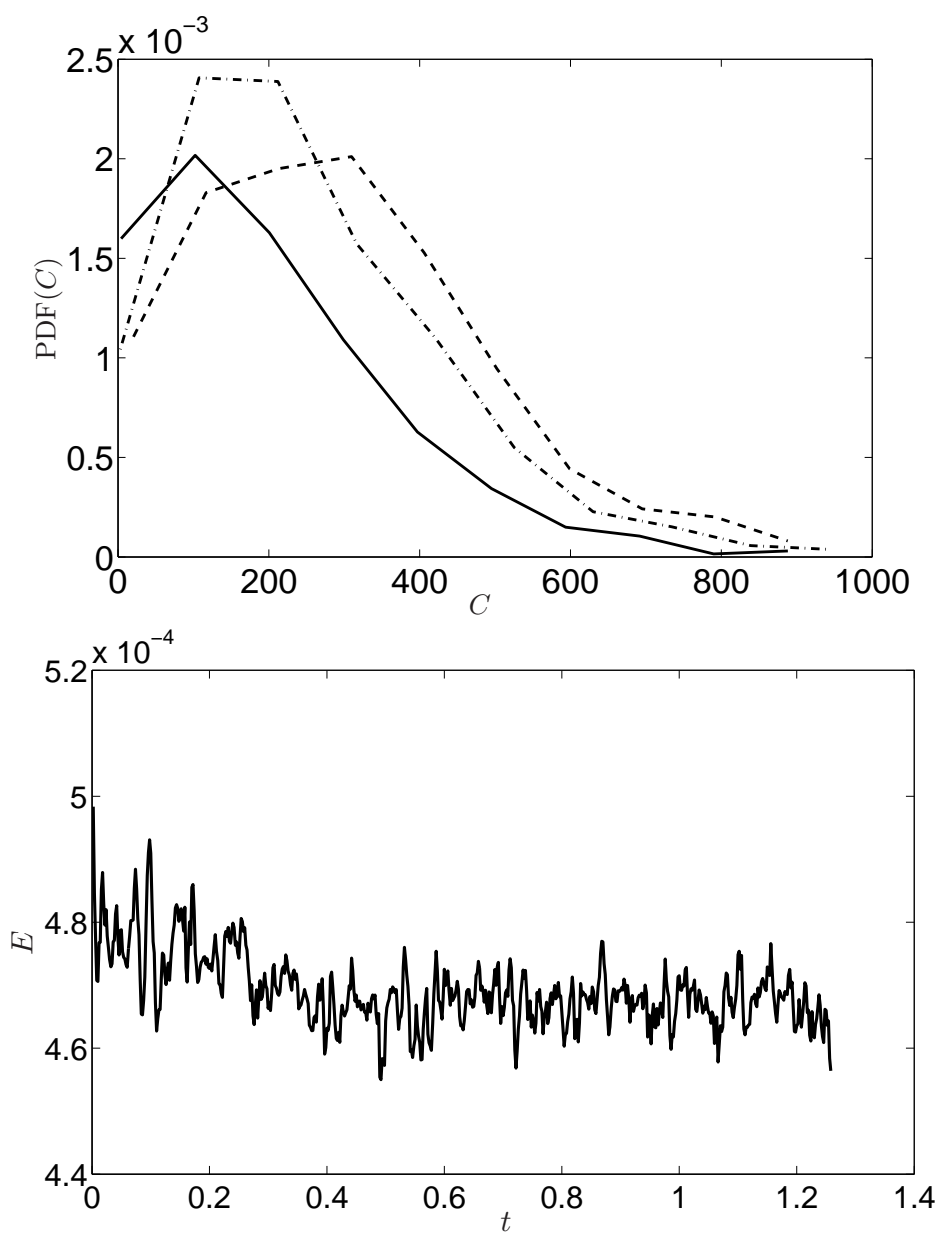

FIG. 21: Top: Probability density function of the curvature $\operatorname{PDF}(C)$ vs $C\left(\mathrm{~cm}^{-1}\right)$ corresponding to Fig. (20) at $t=0$ (solid line), $t=0.2 \mathrm{~s}$ (dashed line) and $t=0.8 \mathrm{~s}$ (dot dashed line) Note that the peak moves to larger values as in Fig. 10 Bottom: Corresponding behaviour of the energy $E\left(\mathrm{~cm}^{2} / \mathrm{s}^{2}\right)$ vs time $t(\mathrm{~s})$. 


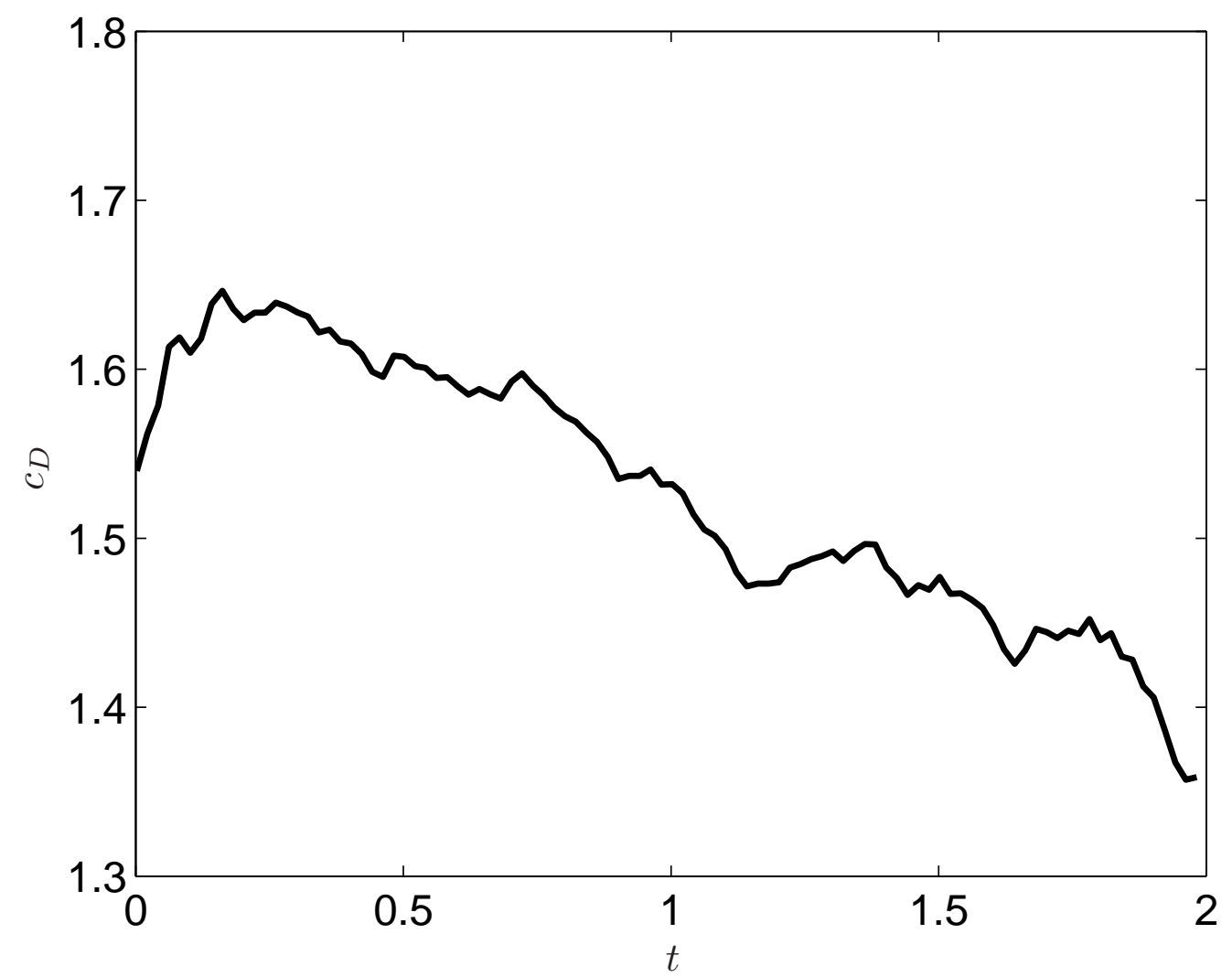

FIG. 22: Correlation dimension $c_{D}$ as a function of time $t(\mathrm{~s})$ corresponding to Fig. 20. Note a rapid increase of $c_{D}$ after the reconnection at $t=0.008 \mathrm{~s}$, and the slow decrease which follows due to numerical dissipation. 